\title{
UICN
}

\section{Statut vert des espèces de I'UICN}

Norme mondiale pour mesurer le rétablissement des espèces et évaluer l'impact de la conservation

Préparé par le Groupe d'étude de la CSE UICN sur l'efficacité de la conservation des espèces Version 2.0 


\section{À propos de l'UICN}

L'UICN est une union unique de Membres composée de gouvernements et d'organisations de la société civile. Elle offre aux organisations publiques, privées et non-gouvernementales les connaissances et les outils nécessaires pour que le progrès humain, le développement économique et la conservation de la nature se réalisent en harmonie.

Créée en 1948, l'UICN est aujourd'hui le réseau environnemental le plus important et le plus diversifié au monde. Elle compte avec l'expérience, les ressources et le poids de ses 1400 organisations Membres et les compétences de plus de 18000 experts. Elle fait autorité en ce qui concerne les données, les évaluations et les analyses en matière de conservation. La diversité de ses Membres font de l'UICN un incubateur et un référentiel fiable pour les bonnes pratiques, les outils et les normes internationales.

L'UICN offre un lieu de débat neutre où diverses parties prenantes, y compris les gouvernements, les ONG, les scientifiques, les entreprises, les communautés locales, les organisations des peuples autochtones et autres peuvent travailler ensemble à l'élaboration et à la mise en oeuvre de solutions aux défis environnementaux et parvenir à un développement durable.

En collaboration avec de nombreux partenaires et supporters, I'UICN met en oeuvre un vaste portefeuille de projets de conservation dans le monde entier. Associant les connaissances scientifiques les plus pointues et le savoir traditionnel des communautés locales, ces projets visent à mettre un terme à la disparition des habitats, à restaurer les écosystèmes et à améliorer le bien-être des populations.

www.iucn.org/fr

https://twitter.com/IUCN/ 
Statut vert des espèces de l'UICN

Norme mondiale pour mesurer le rétablissement des espèces et évaluer l'impact de la conservation

Préparé par le Groupe d'étude de la CSE UICN sur l'efficacité de la conservation des espèces Version 2.0 
La terminologie géographique employée dans cet ouvrage, de même que sa présentation, ne sont en aucune manière l'expression d'une opinion quelconque de la part de l'UICN ou des autres organisations concernées sur le statut juridique ou l'autorité de quelque pays, territoire ou région que ce soit, ou sur la délimitation de ses frontières.

Les opinions exprimées dans cette publication ne reflètent pas nécessairement celles de l'UICN ou des autres organisations concernées.

Document préparé par le Groupe d'étude de la CSE UICN sur l'efficacité de la conservation des espèces sur l'efficacité de la conservation des espèces. Approuvé lors de la 102nd réunion du Conseil de l'UICN, Gland, Suisse (12/01/2020).

L'UICN remercie ses partenaires cadre pour leur précieux support et tout particulièrement : le Ministère des affaires étrangères du Danemark ; le Ministère des affaires étrangères de la Finlande ; le Gouvernement Français et l'Agence Française de Développement (AFD) ; le Ministère de l'environnement de la République de Corée ; l'Agence norvégienne de développement et de coopération (Norad) ; l'Agence suédoise de coopération internationale au développement (Asdi) ; la Direction du développement et de la coopération de la Suisse (DDC) et le Département d'Etat des Etats Unis d'Amérique.

L'UICN et les autres organisations concernées déclinent toute responsabilité en cas d'erreurs ou d'omissions intervenues lors de la traduction en français de ce document dont la version originale est en anglais. En cas de divergences, veuillez vous référer à l'édition originale : IUCN Green Status of Species: A global standard for measuring species recovery and assessing conservation impact. Version 2.0. Publié par : UICN, Gland, Suisse. DOI : https://doi.org/10.2305/IUCN.CH.2021.02.en

Le présent ouvrage a pu être publié grâce à un soutien financier du Natural Environment Research Council (NERC) de l'UKRI, du WWF US, de Re:wild (formellement GWC), de la Commission pour la sauvegarde des espèces des espèces de l'UICN, de l'Université d'Oxford, de l'Université de Stony Brook et de la Fondation Prince Albert II de Monaco (par l'intermédiaire de la Cambridge Conservation Initiative).

Publié par: $\quad$ UICN, Gland, Suisse

Produit par: le Groupe d'étude de la CSE UICN sur l'efficacité de la conservation des espèces

Droits d'auteur : (c) $2021 \mathrm{UICN}$, Union internationale pour la conservation de la nature et de ses ressources La reproduction de cette publication à des fins non commerciales, notamment éducatives, est permise sans autorisation écrite préalable des détenteurs des droits d'auteur à condition que la source soit dûment citée. La reproduction de cette publication à des fins commerciales, notamment en vue de la vente, est interdite sans autorisation écrite préalable des détenteurs des droits d'auteur.

Citation UICN (2021). Statut vert des espèces de l'UICN : Norme mondiale pour mesurer le rétablissement des espèces et évaluer recommandée: l'impact de la conservation. Version 2.0. Gland, Suisse: UICN.

ISBN: 978-2-8317-2125-5 (PDF)

DOI: https://doi.org/10.2305/IUCN.CH.2021.02.fr

Photo de couverture : [couverture] Rainette patte d'oie, Hypsiboas boans, dans la Réserve naturelle de Cocobolo au Panama @ Robin Moore [dos] Magnolia orbiculata au Jardin botanique national de Cuba @ Ernesto Teste

Mise en page : Jessica Avanidhar, jessavanidhar.com

Traduit par: $\quad$ Alexa Dubreuil-Storer (IDFP Translation Services), Royaume-Uni 


\section{Table des Matières}

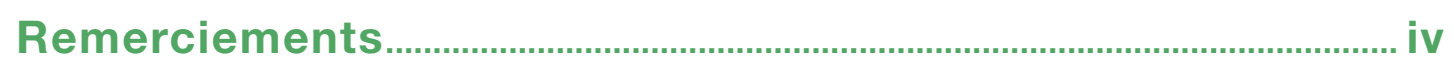

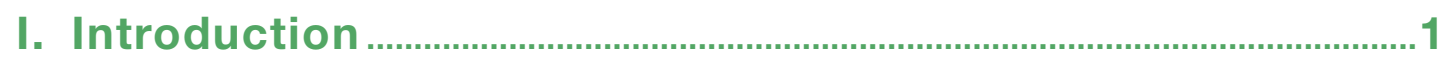

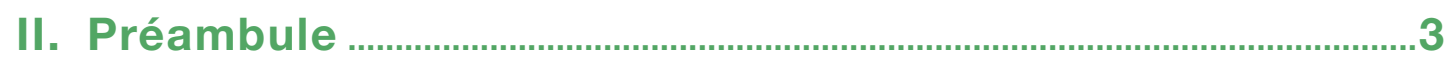

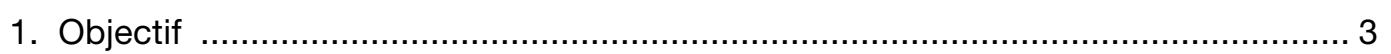

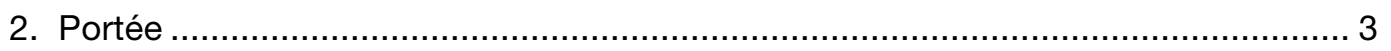

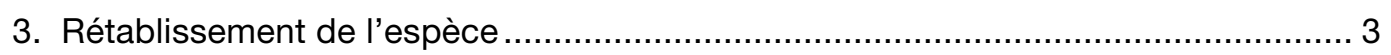

4. Lien avec la Liste rouge de I'UICN ..................................................................... 4

5. Lien avec la planification et les priorités en matière de conservation ...................... 4

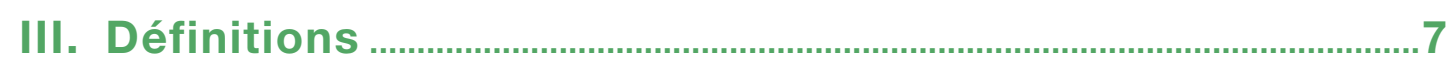

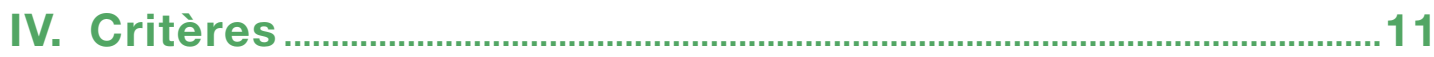

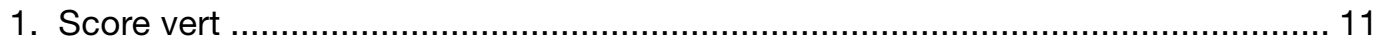

2. Paramètres d'impact de la conservation.............................................................. 12

3. Catégories et seuils ............................................................................. 13

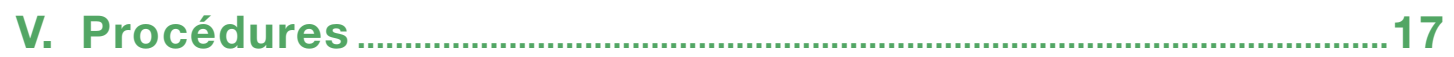

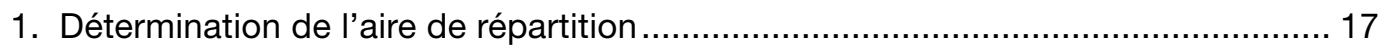

2. Délimitation des unités spatiales................................................................... 18

3. Évaluation de l'état dans une unité spatiale .......................................................... 19

4. Élaboration du scénario Actuel contrefactuel.................................................... 20

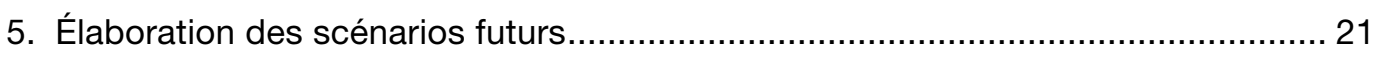

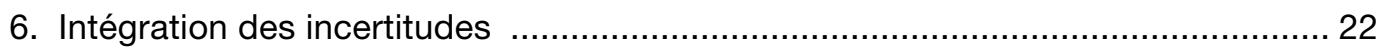

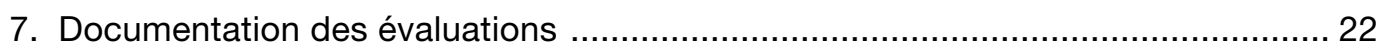

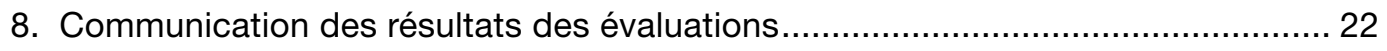

9. Évaluations régionales (dont les évaluations nationales) ...................................... 22

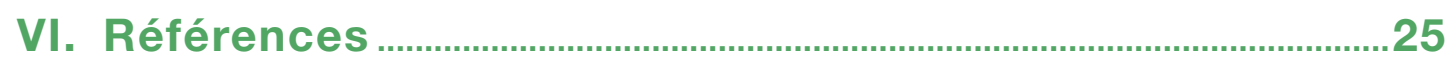




\section{Remerciements}

L'UICN tient à remercier le Groupe d'étude de l'UICN sur l'efficacité de la conservation des espèces (en anglais « Species Conservation Success Task Force » ou SCSTF) pour son dévouement et ses efforts en vue de proposer les méthodes du Statut vert des espèces de l'UICN et de tester rigoureusement les méthodes proposées par le biais de consultations, d'ateliers et d'études scientifiques. Le SCSTF était coprésidé par Barney Long et Elizabeth Bennett, et coordonné par Molly Grace. Les membres du groupe principal du SCSTF étaient : Resit Akçakaya, Craig Hilton-Taylor, Mike Hoffmann, E.J. Milner-Gulland, Richard Jenkins, Ana Nieto et Richard Young; et les membres du groupe élargi du SCSTF étaient : Thomas Brooks, Anna Heath, Simon Hedges, David Keith, David Mallon, Erik Meijaard, Ana Rodrigues, Jon Paul Rodríguez, PJ Stephenson et Simon Stuart. Nous remercions tout particulièrement Resit Akçakaya, qui a joué un rôle prépondérant dans le développement scientifique de la méthode.

Pour élaborer la méthode du Statut vert des espèces, le SCSTF a constitué plusieurs groupes de travail, comprenant à la fois des membres du SCSTF et des experts externes : un Groupe de travail sur les cibles et les références, dirigé par PJ Stephenson ; un Groupe de travail sur les scénarios, dirigé par E.J. Milner-Gulland ; un Groupe de travail sur la viabilité et la fonctionnalité, dirigé par Resit Akçakaya ; un Groupe de travail sur les unités spatiales, dirigé par Elizabeth Bennett et Molly Grace ; un Groupe de travail sur les questions techniques, dirigé par Craig Hilton-Taylor et Michael Hoffmann ; et un Groupe de travail sur la communication, dirigé par Barney Long. Les personnes non membres du SCSTF ayant participé à ces groupes étaient : Alison Boyer, Joe Bull, Onnie Byers, Cheli Cresswell Sinclair, Axel Hochkirch, Ackbar Joolia, Phil McGowan, Elaine Paterson, Eric Sanderson, Carrie Stengel, Ricardo Tejeda, Jessica Welch et le Groupe de travail technique de la Liste rouge.

Le SCSTF a mené un vaste projet pilote sur la méthode du Statut vert des espèces portant sur 181 espèces, sous la direction de Molly Grace. Plus de 200 membres du Groupe de spécialistes de la CSE ainsi que des experts indépendants en espèces ont offert leur temps et leurs connaissances pour mener des évaluations pilotes des espèces, lesquelles étaient indispensables pour affiner la méthode. Quinze de ces évaluations ont été commanditées par National Geographic et dirigées par PJ Stephenson. Nigel Dudley et Hannah Timmins (Equilibrium Research) ont mené une consultation auprès des utilisateurs finaux potentiels ; les commentaires des représentants des entreprises, des politiques, des ONG et d'autres intervenants ont contribué à rationaliser la méthode. Sarah Leonard a mené une consultation terminologique avec les bureaux régionaux de l'UICN, les représentants régionaux de la CSE/UICN, les zoos et aquariums et les journalistes, afin de permettre une communication efficace sur le produit final.

Les travaux du SCSTF ont pu avoir lieu grâce au généreux soutien financier du Natural Environment Research Council (NERC) de l'UKRI, du WWF US, de Re:wild (formellement GWC), de la Commission pour la sauvegarde des espèces de I'UICN, de l'Université d'Oxford, de l'Université de Stony Brook et de la Fondation Prince Albert II de Monaco (par l'intermédiaire de la Cambridge Conservation Initiative). Nous remercions les organismes suivants pour leur soutien financier dans le cadre des ateliers du SCSTF : le NERC, la Fondation Franklinia, la Fondation Prince Albert II de Monaco (par l'intermédiaire de la Cambridge Conservation Initiative) et le Knowledge Exchange Seed Fund (fonds pour l'échange de connaissances) d'Oxford. Plusieurs organisations ont apporté leur concours en permettant aux membres du SCSTF de contribuer à ces travaux sur leur temps de travail salarié : Wildlife Conservation Society, la Zoological Society of London et Durrell.

Enfin, I'UICN est redevable aux membres des Commissions et aux représentants des organisations membres qui ont participé aux ateliers de consultation ou fourni des commentaires et des suggestions lors du processus d'examen de la présente norme. Collectivement, ces contributions ont permis d'obtenir un système beaucoup plus robuste, convivial et applicable largement. 
7.

text

$=x+\frac{1}{20}$

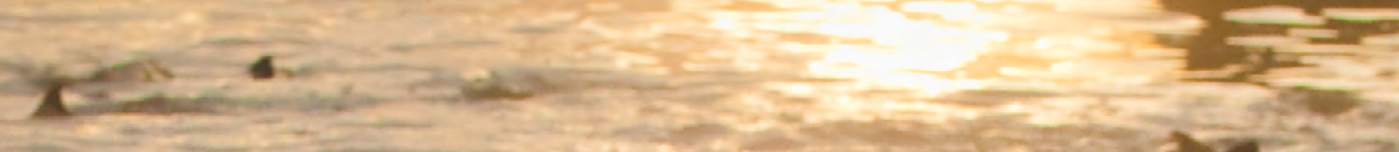

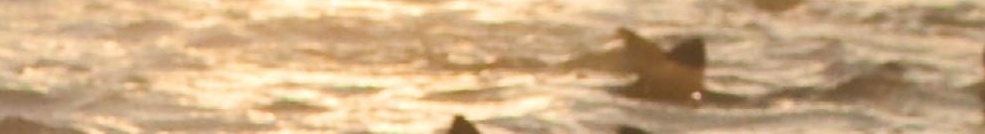

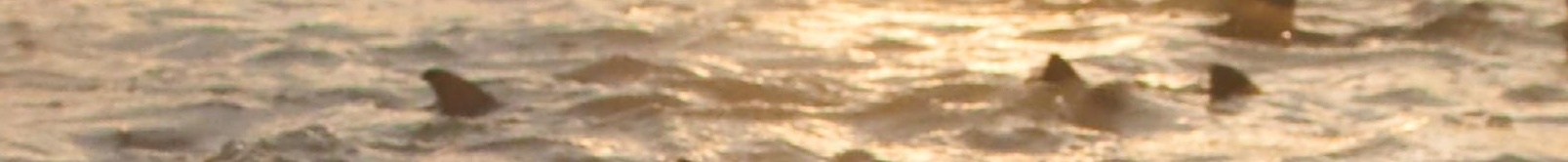
$x_{2}=5$

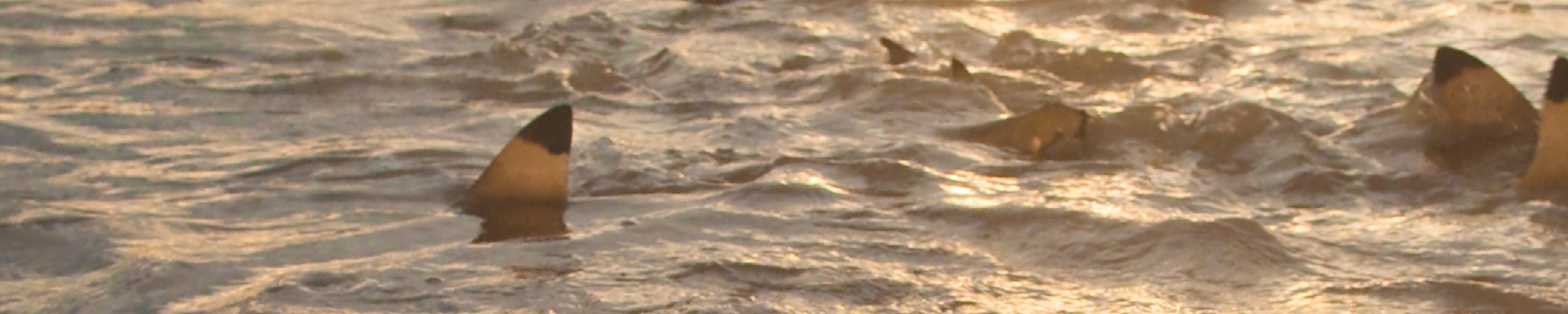

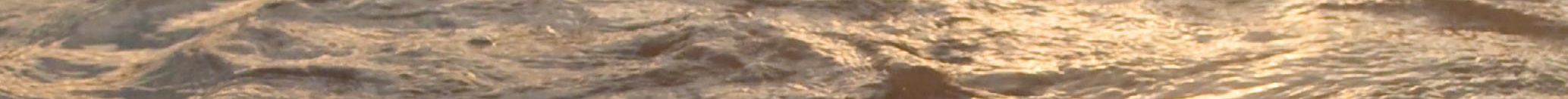

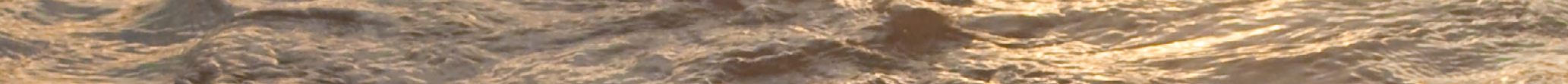

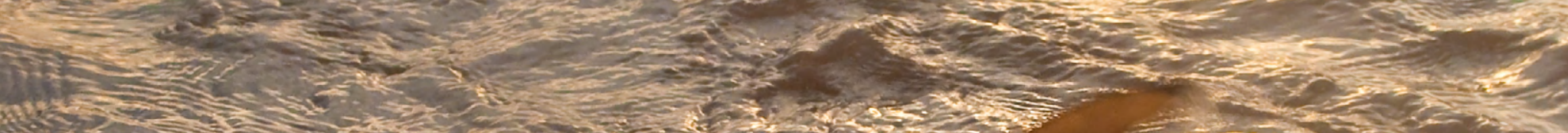

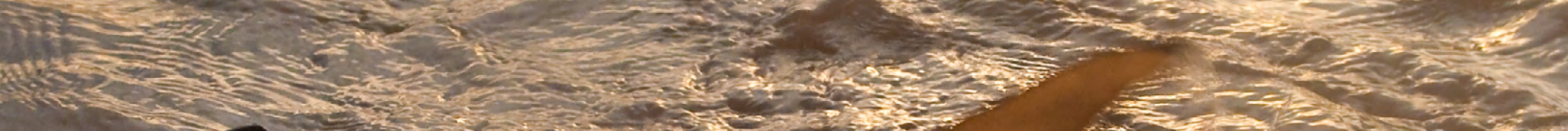

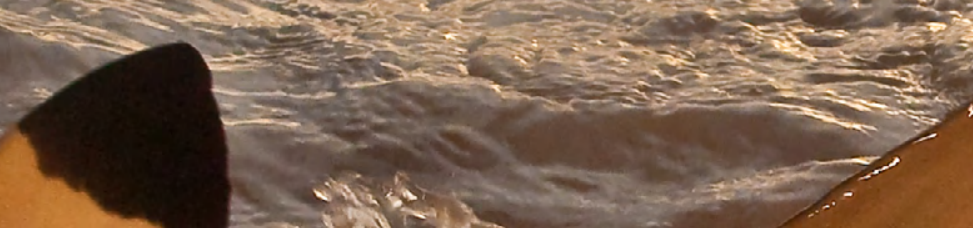

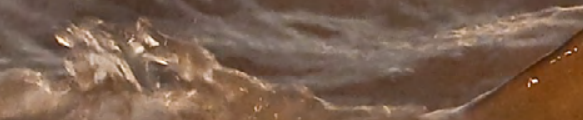

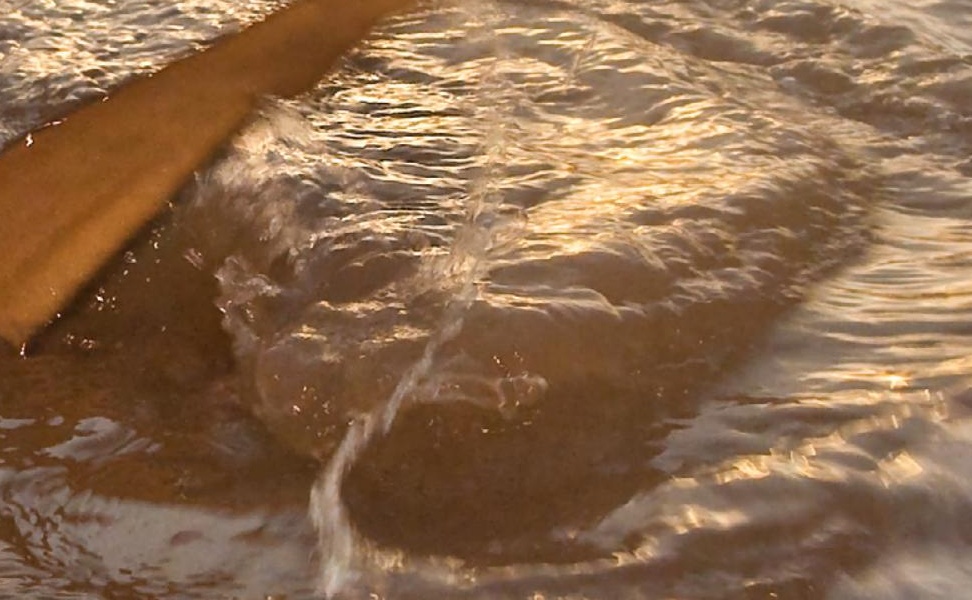

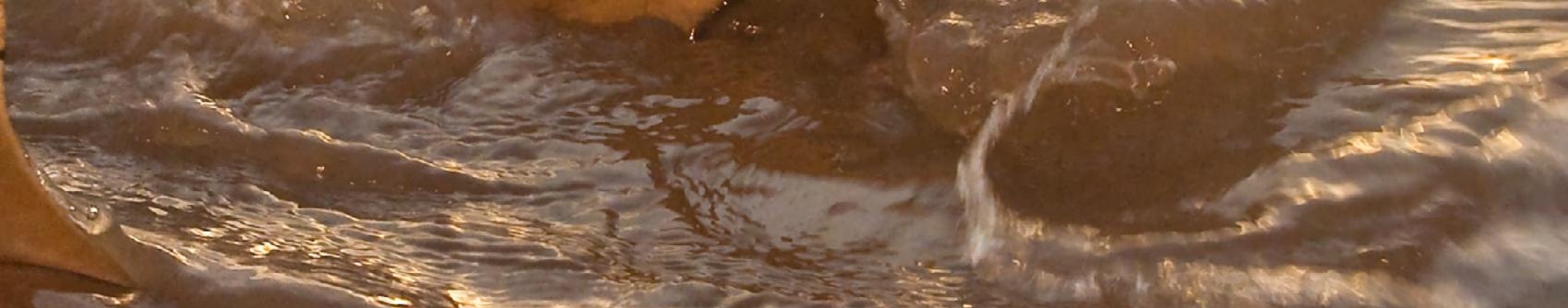

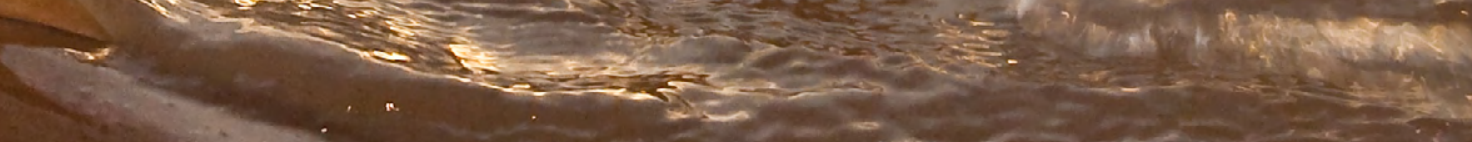
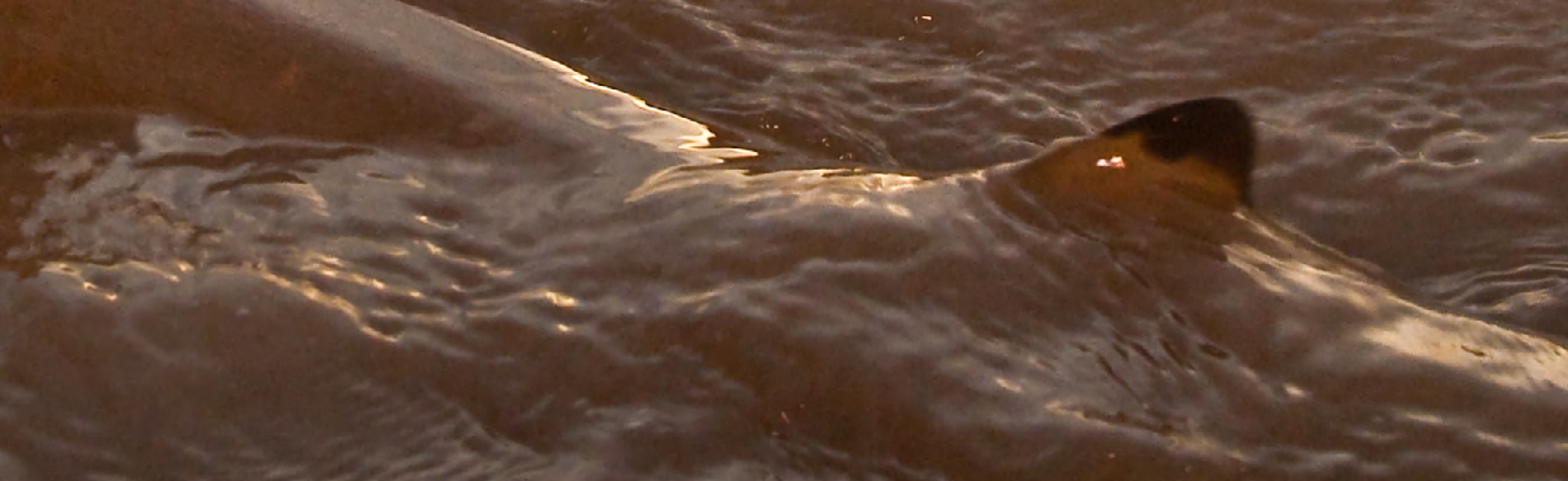

$3 \times$ 

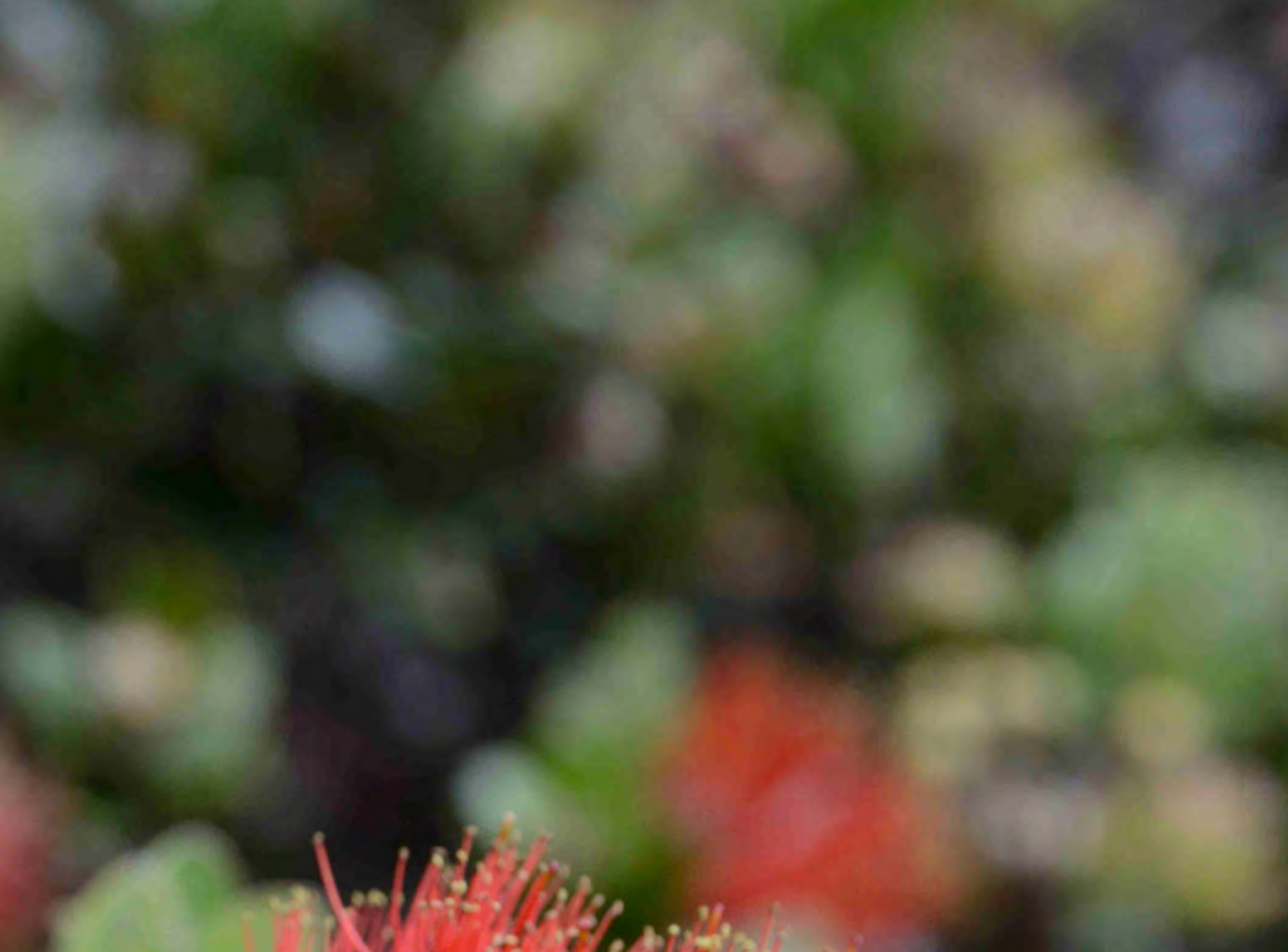

(1)

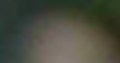

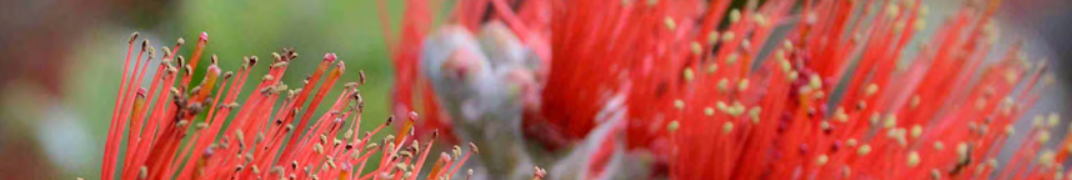

(1)

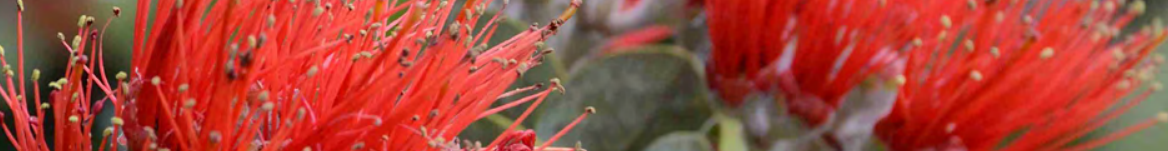
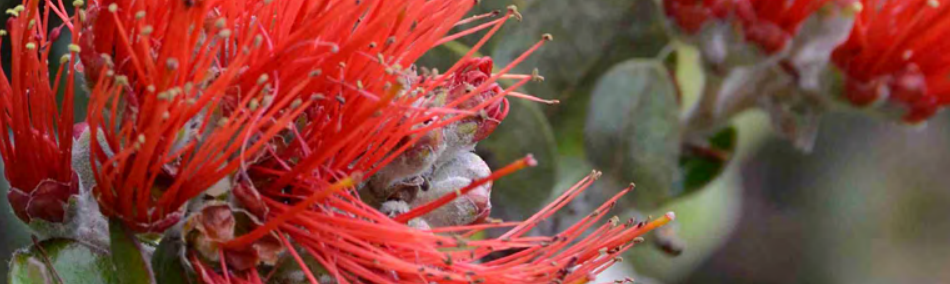
Mes
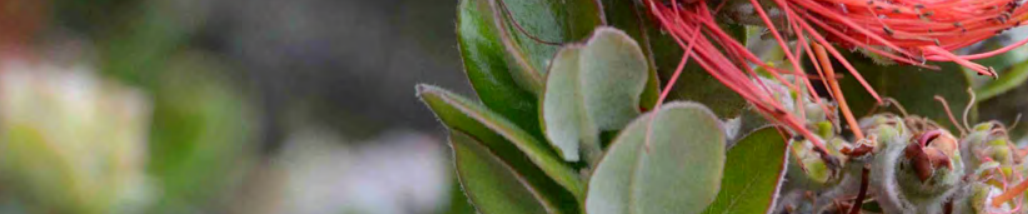

$6+102$
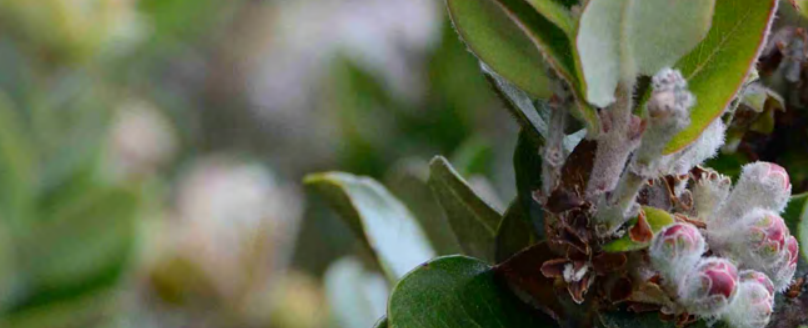

a os 1302 $8=2$

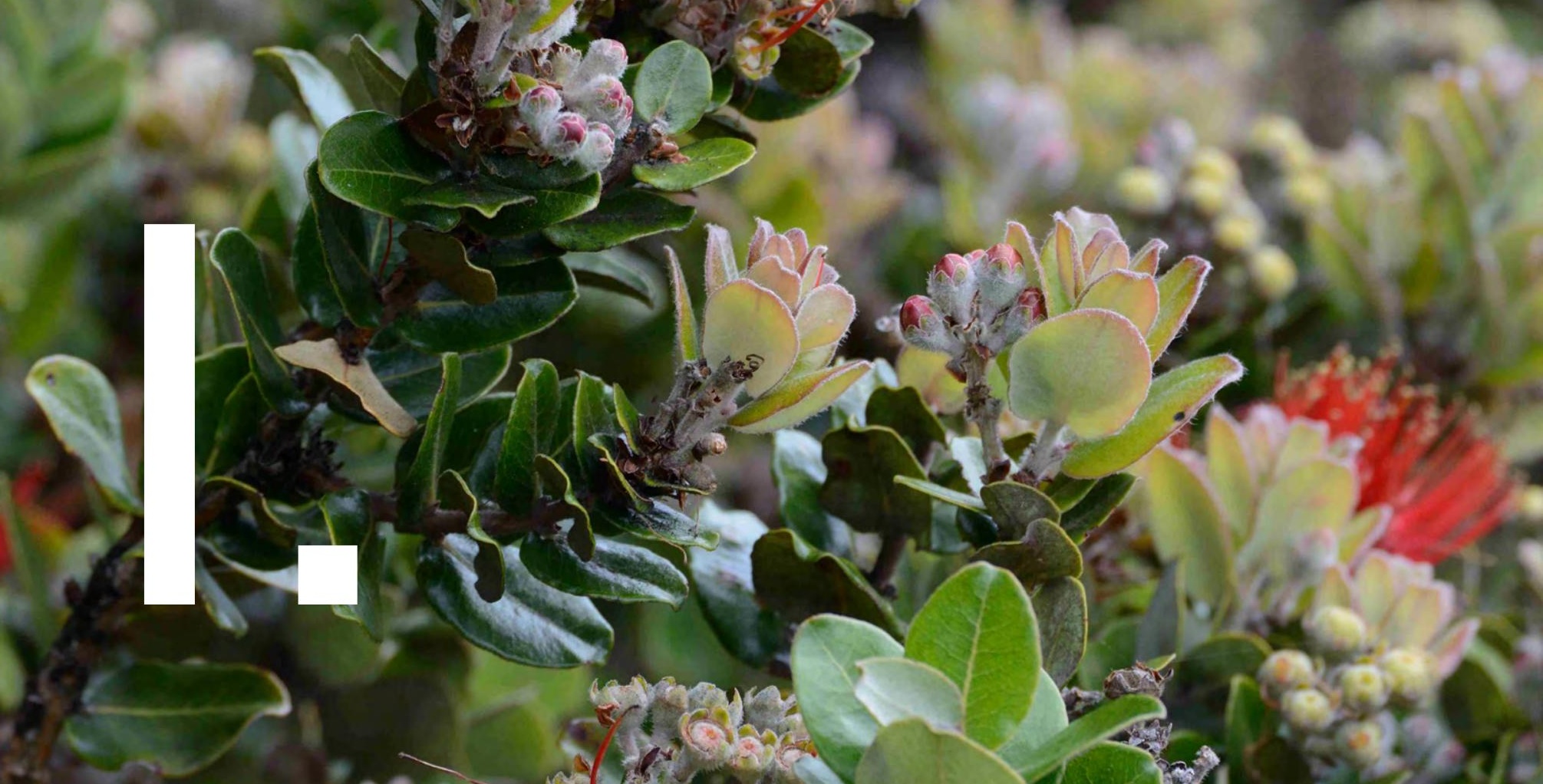

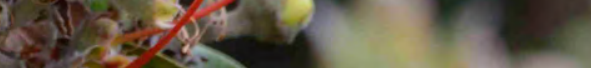

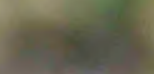

$$
\text { (5) }
$$




\section{Introduction}

Le déclin de nombreuses espèces en voie d'extinction a largement orienté les efforts de conservation de manière à ce que ces espèces continuent d'exister. Toutefois, les conservationnistes reconnaissent depuis longtemps la nécessité de compléter ces efforts par des mesures de reconstitution des populations en déclin dans l'ensemble de l'aire de répartition d'une espèce, et de restauration des espèces dans les écosystèmes où elles ont disparu.

Reconnaissant ce besoin, l'Union internationale pour la conservation de la nature (UICN) a appelé à l'élaboration de critères objectifs pour une Liste verte des espèces, des écosystèmes et des aires protégées (IUCN \& WCPA, 2017). Dans sa résolution WCC-2012-RES-41, le Congrès mondial de la nature de 2012 a prié la « Commission pour la sauvegarde des espèces (CSE) [...] de conduire des consultations scientifiques internationales en vue de développer, pour les listes vertes, des critères objectifs, transparents, reproductibles qui mesurent systématiquement les succès de la conservation des espèces et des écosystèmes ".

En réponse à cela, la Commission pour la sauvegarde des espèces a constitué un Groupe d'étude pour évaluer l'efficacité de la conservation, sous les auspices du Comité de la Liste rouge de l'UICN pour superviser l'élaboration de cette norme. Le Groupe d'étude a élaboré un cadre pour mesurer le rétablissement des espèces et l'impact de la conservation (Akçakaya et al., 2018)', lequel a proposé une définition d'une espèce entièrement rétablie fondée sur la viabilité, la fonctionnalité et la représentation, et a défini quatre paramètres pour quantifier l'importance de la conservation pour une espèce. Ce cadre a été testé sur une série de taxons entre 2018 et 2021 pour garantir une large applicabilité, et les modifications apportées ont été prises en compte dans la présente norme.

Pour bien comprendre les évaluations du Statut vert des espèces de l'UICN, il est très important de se référer aux dernières versions de tous les documents suivants :

1. Contexte et lignes directrices pour le Statut vert des espèces de l'UICN, document qui accompagne la présente norme et qui sera mis à jour périodiquement (ci-après " Contexte et lignes directrices ") ;

2. Catégories et Critères de la Liste rouge de I'UICN (UICN, 2012a) ;

3. Lignes directrices pour l'utilisation des Catégories et Critères de la Liste rouge de l'UICN (UICN, 2019) ;

4. Lignes directrices pour l'application des Critères de la Liste rouge de l'UICN aux niveaux régional et national (UICN, 2012b).

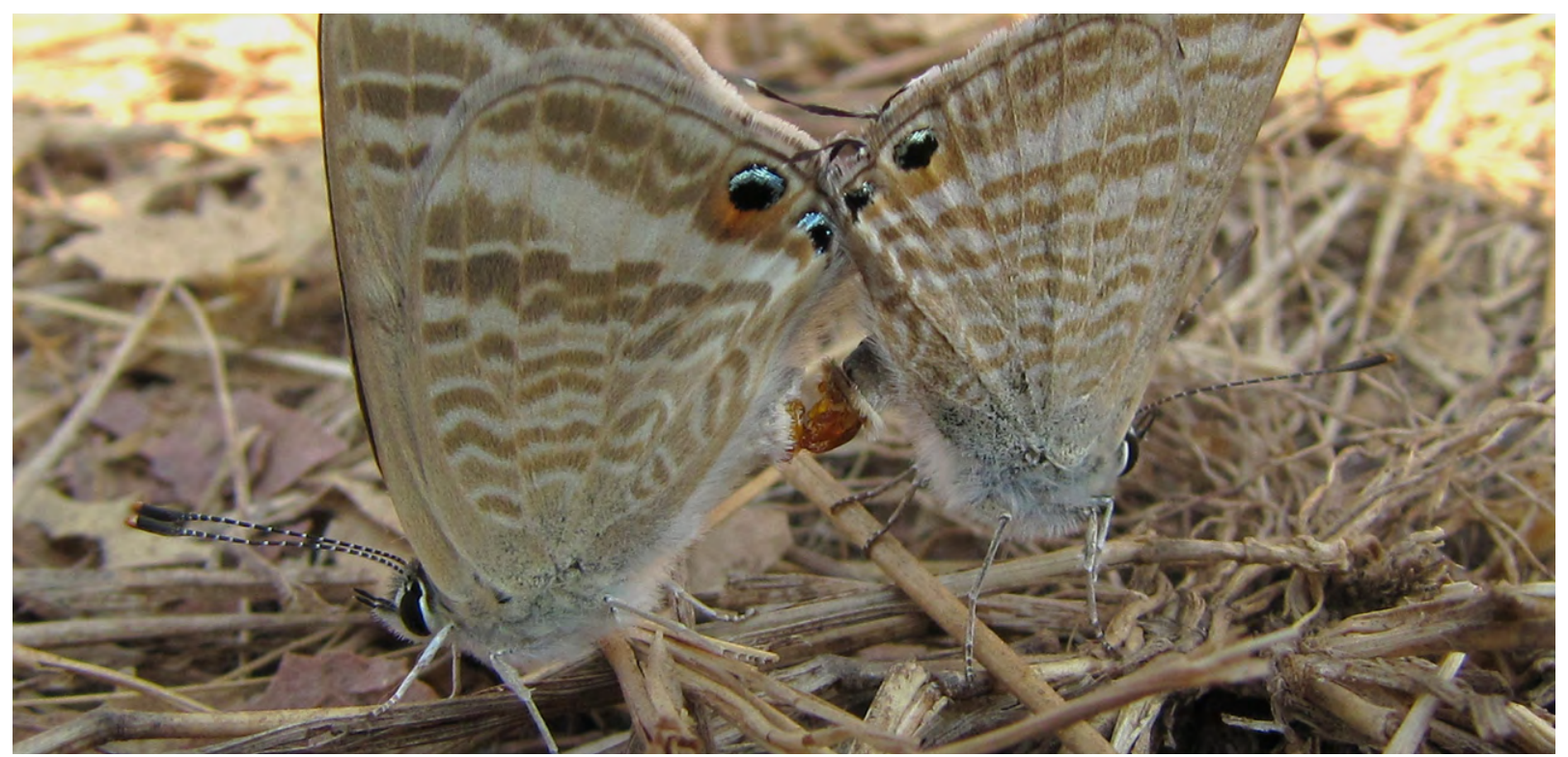

Accouplement d'azurés porte-queue (Lampides boeticus) @ Neha Mujumdar

1 La publication Akçakaya et al. (2018) est considérée comme la version 1.0 de la présente norme ; le document que vous lisez est la version 2.0, laquelle tient compte des modifications apportées suite aux tests et aux consultations qui ont eu lieu entre 2018 et 2020. 


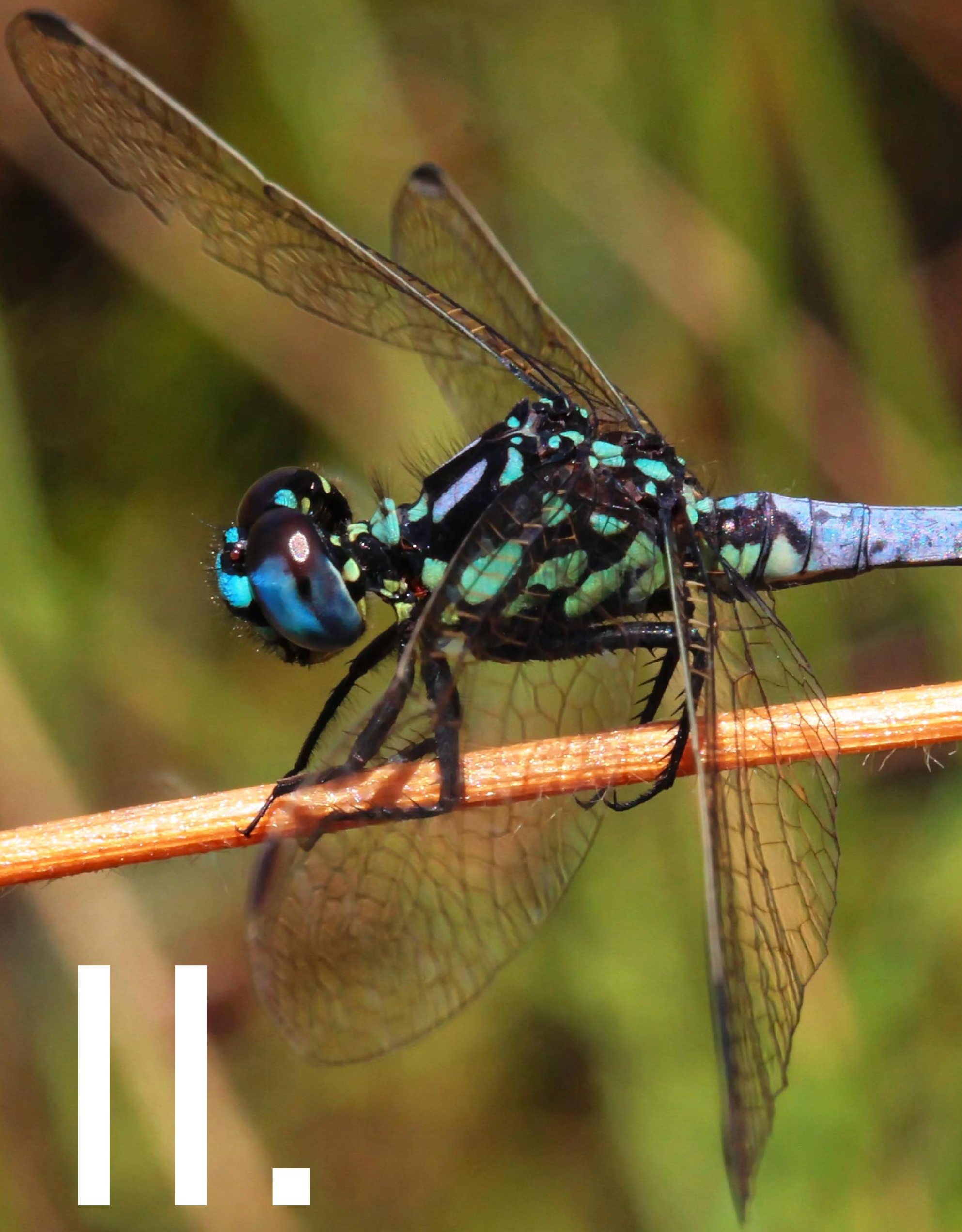




\section{Préambule}

\section{Objectif}

Le Statut vert des espèces de I'UICN comprend cinq objectifs principaux :

i. Fournir un cadre normalisé pour mesurer le rétablissement des espèces ;

ii. Reconnaître les réalisations en matière de conservation ;

iii. Mettre en évidence les espèces dont le statut de conservation actuel dépend de la poursuite des actions de conservation ;

iv. Prévoir l'impact attendu des actions de conservation planifiées ; et

v. Relever les niveaux d'ambition pour le rétablissement des espèces à long terme.

Collectivement, ces objectifs encouragent la conservation en vue du rétablissement des espèces, dans l'ensemble de l'aire de répartition de ces espèces. Ils sont représentés par un Score de rétablissement de l'espèce et par quatre paramètres d'impact de la conservation (Héritage de la conservation, Dépendance envers la conservation, Gain par conservation, Potentiel de rétablissement), lesquels sont quantifiés en tant que différences entre les Scores verts de l'espèce à différentes étapes temporelles ou selon différents scénarios.

\section{Portée}

Les définitions et les paramètres du Statut vert des espèces peuvent être appliqués à toutes les espèces, à l'exception des micro-organismes. Les espèces peuvent être évaluées dans le cadre de ce protocole, quelle que soit leur catégorie de la Liste rouge et qu'elles aient fait l'objet de mesures de conservation ou non. Pour des raisons pratiques (voir section V.3), le Statut vert d'une espèce doit être évalué après, ou en même temps que, l'évaluation de la Liste rouge de cette espèce.

\section{Rétablissement de l'espèce}

Dans la présente norme, la définition d'une espèce connaissant un « Rétablissement complet » se base sur la viabilité, la fonctionnalité et la représentation (voir définition à la section III). La viabilité est la première exigence essentielle mais elle n'est pas suffisante pour reconnaître une espèce comme rétablie. Pour être considérée comme entièrement rétablie, une espèce doit aussi présenter les interactions écologiques, fonctions et autres rôles écosystémiques qui la caractérisent, et être présente dans un ensemble représentatif d'écosystèmes et de communautés dans toute son aire de répartition. Les aspects relatifs à la viabilité et à la fonctionnalité sont traités lors de l'évaluation de l'état de la population de l'espèce dans chaque unité spatiale (voir sections IV.1, V.3.c et V.3.d), et la notion de représentation est traitée en réalisant l'évaluation dans toutes les unités spatiales de l'aire de répartition de l'espèce (voir sections IV.1 et V.2). La définition fondée sur ces caractéristiques est utilisée pour mesurer le rétablissement d'une espèce, lequel est exprimé sous forme de Score vert et sert ensuite à définir quatre paramètres d'impact de la conservation afin de quantifier l'importance de la conservation pour l'espèce (voir section III, Définitions).

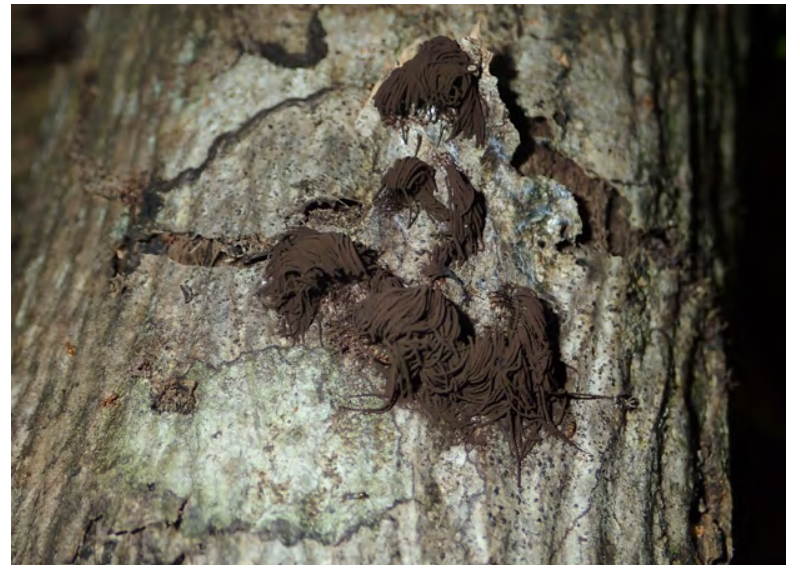

Stemonaria longa en Guadeloupe ( Alain Michaud

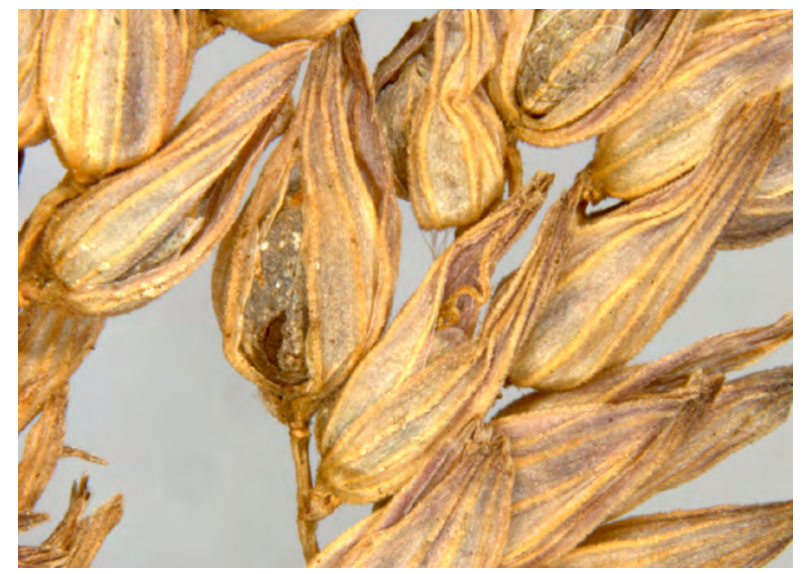

Ustilago suddiana ( C. M. Denchev 


\section{Lien avec la Liste rouge de l'UICN}

Les évaluations du Statut vert ne constituent pas une alternative aux évaluations du risque d'extinction par le biais de la Liste rouge de l'UICN, mais elles fournissent des informations complémentaires. Les résultats d'une évaluation du Statut vert (Score de rétablissement de l'espèce, Catégorie de rétablissement de l'espèce, paramètres d'impact de la conservation et catégories d'impact de la conservation) devraient être considérés conjointement avec la catégorie de la Liste rouge de l'UICN concernant l'espèce. II n'existe pas de relation simple et générale entre le statut figurant sur la Liste rouge et le Statut vert des espèces. Les espèces qui se sont rétablies peuvent encore être menacées ; les espèces qui ne se sont pas rétablies peuvent ne pas être menacées ; et les espèces dont les paramètres d'impact de la conservation sont élevés peuvent ou non être menacées d'extinction. La Liste rouge et le Statut vert fournissent des évaluations distinctes mais liées et complémentaires sur le statut de conservation d'une espèce.

Certains points pertinents pour les évaluations du Statut vert (par exemple les termes utilisés pour définir la viabilité) sont abordés dans les Lignes directrices pour la Liste rouge ; il est donc conseillé aux évaluateurs de consulter également la dernière version des Lignes directrices pour l'utilisation des Catégories et Critères de la Liste rouge de l'UICN (Comité des normes et des pétitions de I'UICN, 2019), car elles sont régulièrement mises à jour.

\section{Lien avec la planification et les priorités en matière de conservation}

Les évaluations du Statut vert n'ont pas vocation à remplacer le processus d'élaboration d'objectifs, de cibles et de finalités en matière de rétablissement des espèces, lequel relève du processus de planification des actions de conservation qui fait participer toutes les parties prenantes concernées et qui est un cadre idéal et adapté pour fixer des objectifs et des priorités de conservation. Au lieu de cela, les évaluations de Statut vert visent à être une partie utile et intégrale de ce processus de planification et à refléter les objectifs et aspirations établis lors de ce processus. Les plans de conservation ou de rétablissement des espèces sont souvent élaborés avec des mesures et des actions de conservation couvrant un horizon temporel relativement court (p. ex. cinq ou dix ans), ce qui se reflète dans le paramètre Gain par conservation. Ces plans sont généralement imbriqués dans une vision à plus long terme, dont la durée peut souvent aller de 50 à 100 ans. Le paramètre Potentiel de rétablissement est aligné sur cette vision à long terme. L'échelle de temps pour le Potentiel de rétablissement est fixée à 100 ans, afin d'établir un lien explicite avec l'énoncé de vision de nombreux plans d'action et stratégies de conservation. Le Potentiel de rétablissement devrait idéalement être fondé sur l'énoncé de vision à long terme d'un processus reconnu de planification d'actions, impliquant un engagement réfléchi et approprié des parties prenantes. Les processus de planification de la conservation s'appuient souvent sur les actions de conservation passées et les évaluations des impacts de la conservation dans différentes parties de l'aire de répartition de l'espèce, ainsi que sur les besoins de conservation à court terme de l'espèce. Ces éléments sont pris en compte dans les paramètres Héritage de la conservation et Dépendance envers la conservation.

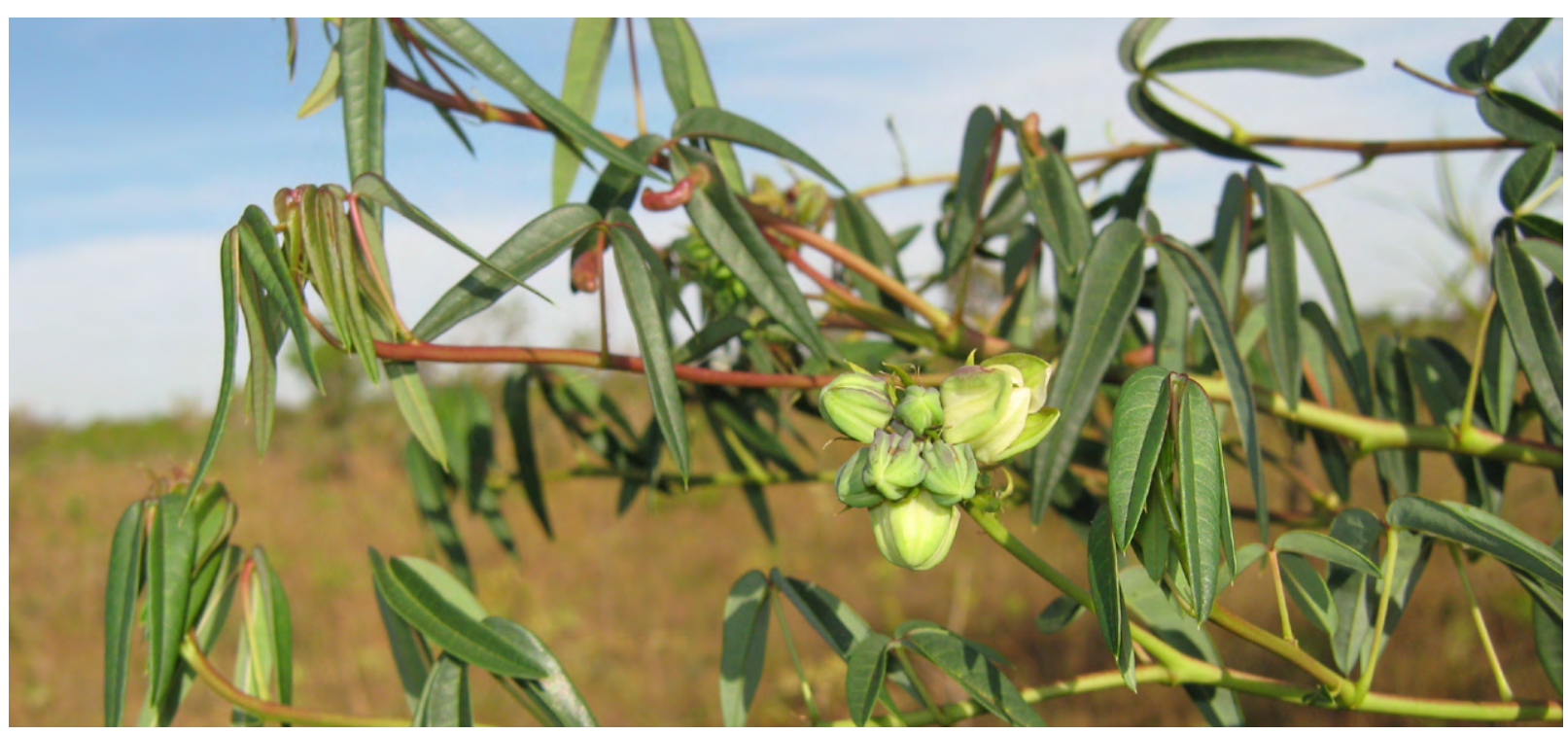

Manihot gracilis Pohl, espèce sauvage apparentée (pool génique secondaire) au manioc (M. esculenta subsp. esculenta), au Brésil (C) Marcelo Simon 


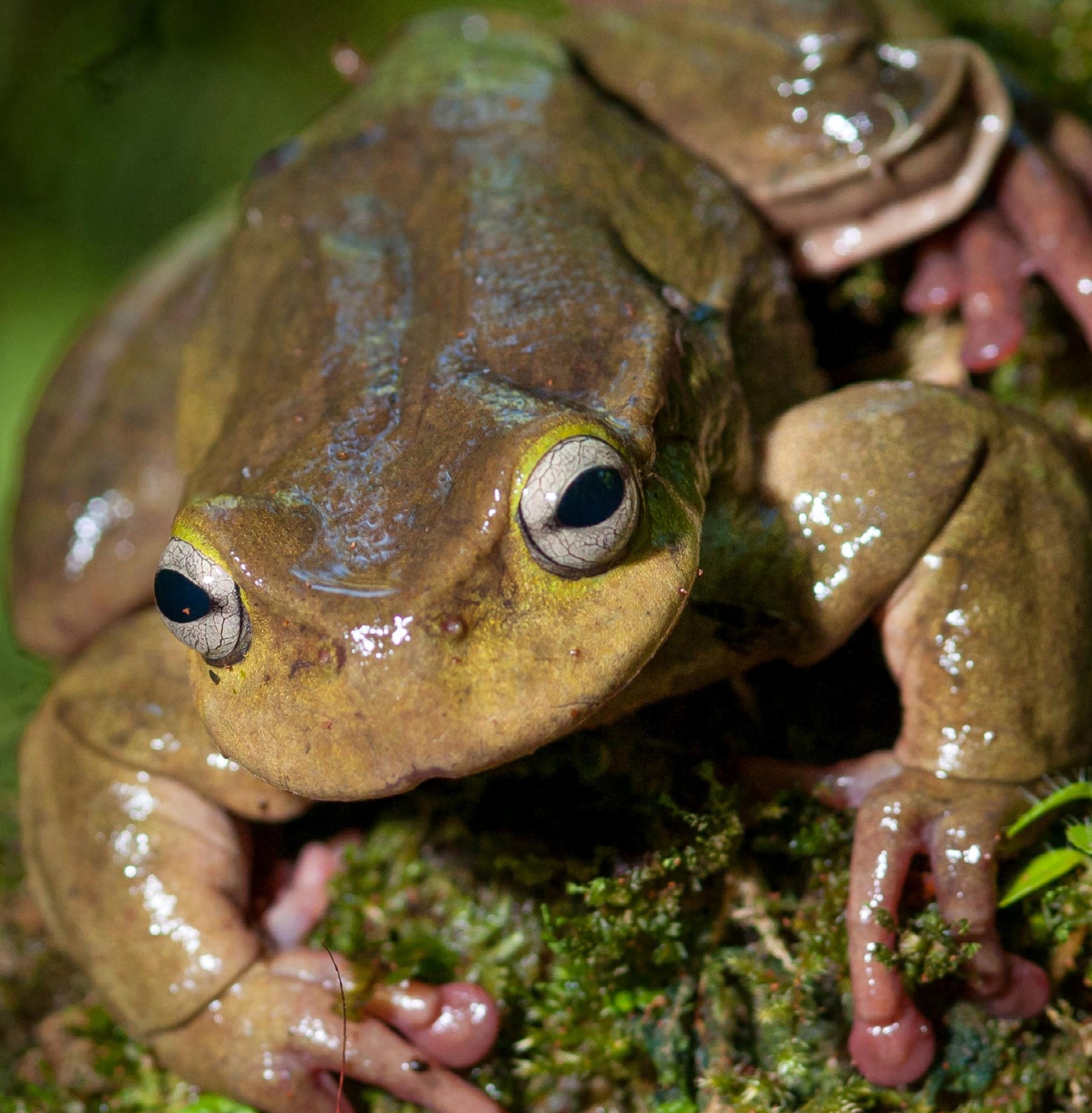

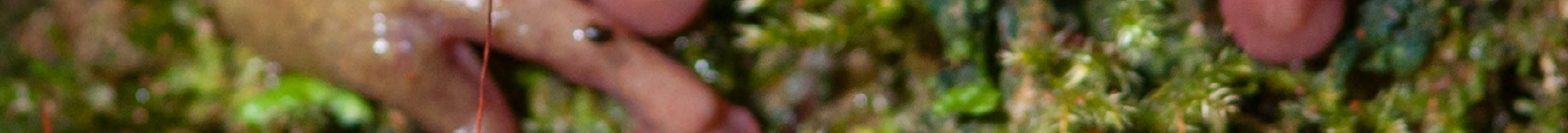
Q.

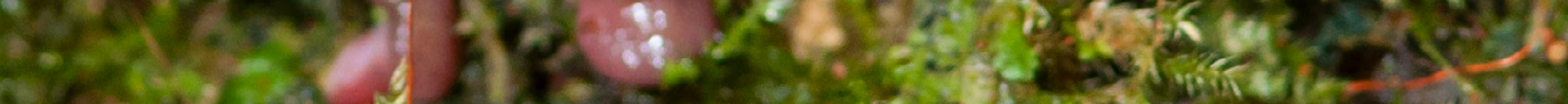

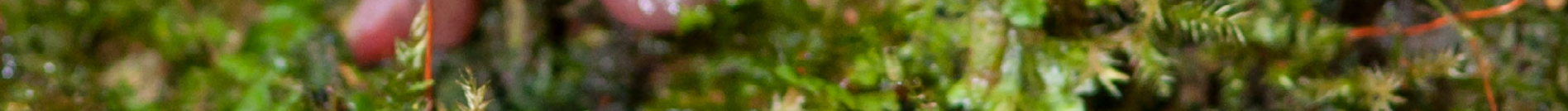
3.20
3

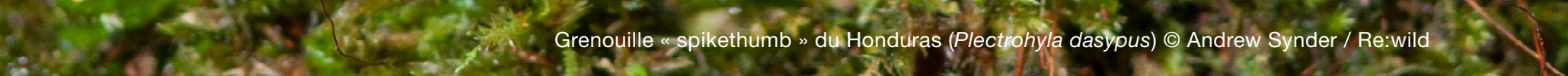

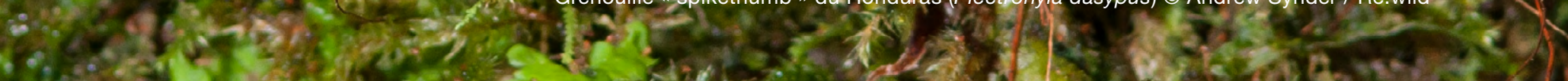

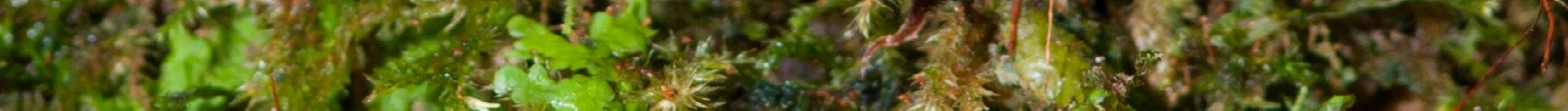



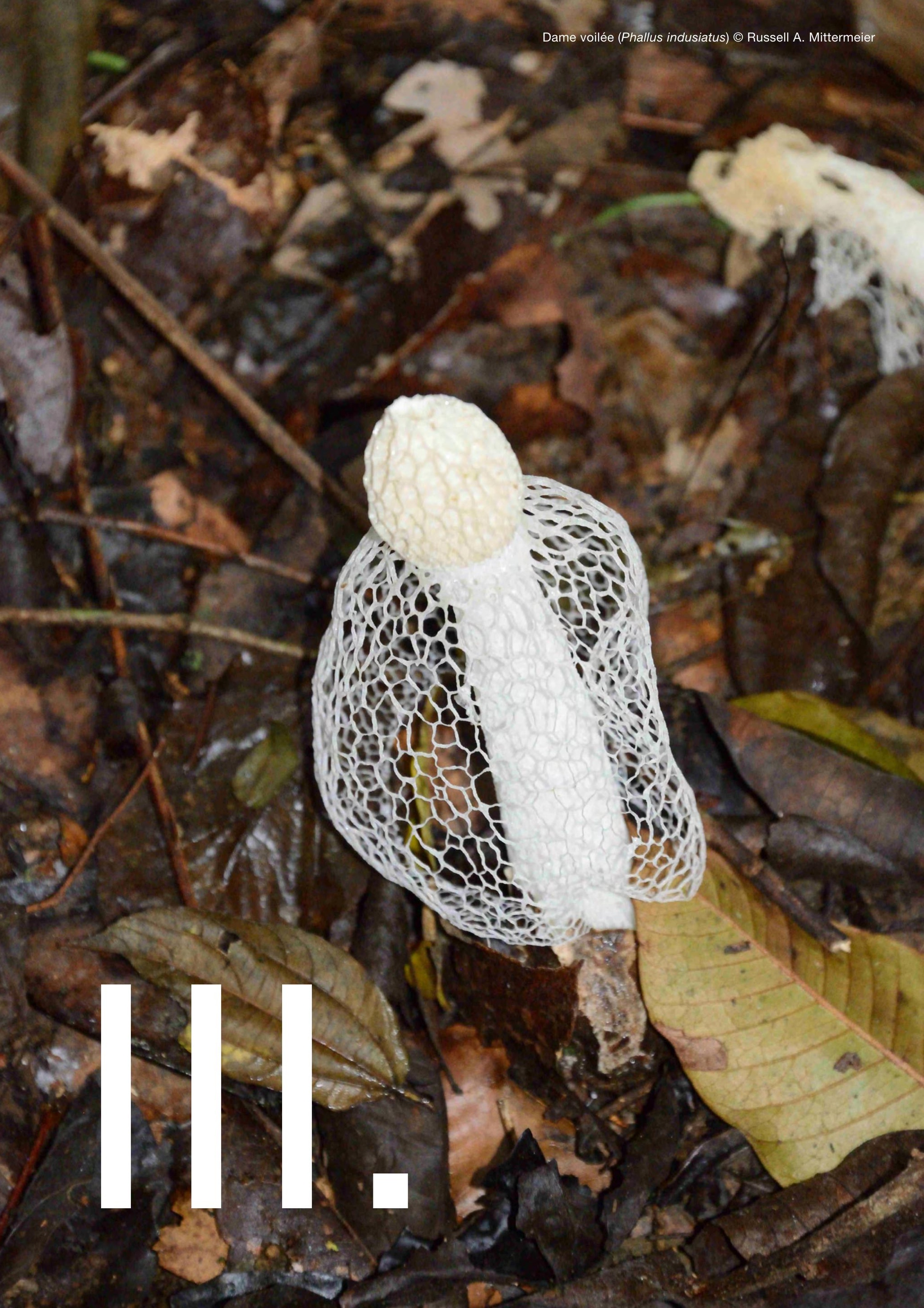


\author{
Absent (état dans une unité \\ spatiale)
}

On dit qu'une espèce est absente d'une unité spatiale donnée si elle n'est pas présente à l'état sauvage, alors que l'unité spatiale fait partie de l'aire de répartition de l'espèce. "Absent " est l'un des quatre états possibles pour la population d'une unité spatiale (les autres étant : Présent, Viable et Fonctionnel).
Toute activité humaine pour laquelle la conservation de la biodiversité est une intention déclarée, même si l'activité n'a pas pour but unique ou principal de conserver la biodiversité, et même si son budget provient de sources autres que les programmes de conservation.
Dépendance envers la conservation
Paramètre d'impact de la conservation qui mesure l'impact des actions de conservation en cours et se définit en tant que modification prévue du Score vert de l'espèce dans un avenir à court terme (10 ans) si toutes les actions de conservation devaient cesser, à compter d'aujourd'hui. Le paramètre est calculé en tant que différence entre le Score vert "Actuel » (ou "Référence actuelle ") et le Score vert "Futur-sans-conservation » (voir section IV.1, IV.2, V.5.c, et Figure 1).

Gain par conservation

Paramètre d'impact de la conservation qui mesure l'impact des actions de conservation en cours et planifiées, et qui se définit en tant que modification prévue du Score vert de l'espèce dans un avenir à court terme (10 ans) si les actions de conservation en cours et planifiées sont mises en œuvre efficacement. Le paramètre est calculé en tant que différence entre le Score vert «Actuel » (ou « Référence actuelle ») et le Score vert « Futur-avec-conservation » (voir section IV.1, IV.2, V.5.c, et Figure 1).

Héritage de la conservation

Paramètre d'impact de la conservation qui mesure l'impact des actions de conservation menées à ce jour, et qui se définit en tant que différence entre les Scores verts «Actuel » et «Actuel contrefactuel » de l'espèce (voir section IV.1 et Figure 1).
Chaque paramètre d'impact de la conservation peut être exprimé en pourcentage ou en utilisant des catégories. Pour chacun des quatre paramètres d'impact de la conservation, les catégories correspondent aux niveaux suivants : Négatif, Nul, Faible, Moyen, Élevé ou Indéterminé (voir section IV.3).
Paramètre d'impact de la conservation
L'une des quatre mesures de l'importance des actions de conservation pour l'espèce : Héritage de la conservation; Dépendance envers la conservation ; Gain par conservation ; et Potentiel de rétablissement. Chaque paramètre est calculé en tant que différence entre deux Scores verts.

Contrefactuel

Scénario hypothétique de ce que le statut de l'espèce aurait été aujourd'hui en l'absence d'actions de conservation passées ; il est utilisé pour déterminer l'état «Actuel contrefactuel » dans chaque unité spatiale et le Score vert «Actuel contrefactuel » (voir section IV.1)

Fonction écologique d'une espèce totalité des interactions de l'espèce, déterminant son influence sur les processus écosystémiques ou sa contribution à ces derniers, ainsi que les modes d'interactions intraspécifiques, de comportement et de dynamique sociale caractérisant cette espèce (voir section V.3.d).

Fonctionnalité écologique d'une population

\section{Aire de répartition supplémentaire attendue}

Mesure dans laquelle la population dans une unité spatiale remplit la (ou les) fonction(s) écologique(s) de l'espèce à un moment et dans un lieu donnés (p. ex., une unité spatiale), ce qui est déterminé par sa taille, sa densité et sa structure démographique (voir section V.3.d).

Zones dont on s'attend fortement à ce qu'elles deviennent adaptées et habitées par l'espèce au cours des 100 prochaines années, en tenant compte des déplacements d'aire de répartition résultant du changement climatique et d'autres processus mondiaux et locaux, ainsi que des transferts de conservation (Comité des normes et des pétitions de l'UICN, 2019). 
Espèce viable et écologiquement fonctionnelle dans chaque partie de son aire de répartition. Le Score vert est défini par rapport à cette condition. Cette définition et les paramètres d'impact de la conservation basés sur celle-ci s'appliquent non seulement aux espèces qui ont déjà décliné, mais aussi aux taxons qui n'ont pas décliné (qu'ils aient ou non fait jusque-là l'objet d'actions de conservation). Toutefois, les taxons qui correspondent à cette définition mais qui n'ont pas bénéficié d'actions de conservation relèvent de la catégorie "Aucune diminution ». Une espèce connaissant un « Rétablissement complet » (ou «Aucune diminution ») a un Score vert de $100 \%$. une unité spatiale)
Fonctionnel (état dans

L'état de la population d'une unité spatiale est dit « Fonctionnel » si cette population remplit la (ou les) fonction(s) écologique(s) de l'espèce ; c'est-à-dire qu'elle possède les attributs (y compris, par exemple, l'abondance, la densité et la structure démographique) qui lui permettent d'interagir avec d'autres espèces, de contribuer aux processus écosystémiques et/ou de présenter les modes d'interactions intraspécifiques, de comportement et de dynamique sociale caractérisant cette espèce. "Fonctionnel » est l'un des quatre états possibles pour la population d'une unité spatiale (les autres étant : Absent, Présent et Viable). Voir section V.3.d et Contexte et lignes directrices section 4.5.

Score vert

Valeur numérique comprise entre $0 \%$ et $100 \%$, représentant le niveau de proximité de l'espèce par rapport à son Rétablissement complet. Une valeur de $0 \%$ signifie que l'espèce est Éteinte ou Éteinte à l'état sauvage, et une valeur de 100 \% signifie qu'elle connaît un Rétablissement complet. Le Score vert est calculé en fonction de l'état (Absent, Présent, Viable et Fonctionnel) dans chaque unité spatiale et peut être calculé pour les périodes passées, actuelles, futures à court terme et futures à long terme, ainsi que pour des scénarios alternatifs (c.-à-d. avec une conservation à différents niveaux [passée, actuelle, planifiée, idéale] ou sans conservation). Ces différents Scores verts sont utilisés pour calculer les paramètres d'impact de la conservation pour chaque espèce, et les espèces sont classées dans des catégories basées sur ces quatre paramètres.
Répartition de l'espèce, générée à partir de données actuelles et historiques (écrites ou orales) ou de preuves physiques de sa présence, en tenant compte de tous les sites d'occurrence connus, déduits ou prévus (UICN, 2012a), y compris les transferts de conservation passés (Comité des normes et des pétitions de I'UICN, 2019), mais sans tenir compte des individus errants. Lorsque les preuves directes sont insuffisantes pour confirmer une occupation précédente, l'existence d'habitats adéquats (d'une proximité écologiquement adaptée par rapport à l'aire de répartition constatée) peut être considérée comme preuve suffisante d'une occupation précédente (CSE/UICN, 2013).

\begin{tabular}{|c|c|}
\hline Parties de l'aire de distribution & Voir « Unité spatiale» \\
\hline $\begin{array}{l}\text { Présent (état dans une } \\
\text { unité spatiale) }\end{array}$ & $\begin{array}{l}\text { L'état de la population d'une unité spatiale est dit «Présent " si l'espèce y } \\
\text { est présente mais que son état n'est pas Viable ou Fonctionnel dans l'unité } \\
\text { spatiale. "Présent » est l'un des quatre états possibles pour la population d'une } \\
\text { unité spatiale (les autres étant : Absent, Viable et Fonctionnel). }\end{array}$ \\
\hline
\end{tabular}

Aire de répartition

Répartition spatiale de l'espèce, comprenant son aire de répartition autochtone et son aire de répartition supplémentaire attendue. Pour le paramètre Héritage de la conservation, la définition de l'aire de répartition est uniquement l'aire de répartition autochtone. Pour les trois autres paramètres d'impact de la conservation, lesquels portent sur l'avenir de l'espèce, l'aire de répartition peut se définir comme étant l'association de son aire de répartition autochtone et de son aire de répartition supplémentaire attendue, selon le moment où l'on estime que l'aire de répartition supplémentaire attendue sera occupée. Voir section V.1.b.

Potentiel de rétablissement

Paramètre d'impact de la conservation qui quantifie l'aspiration ou l'ambition en matière de conservation, et qui se définit comme étant l'amélioration maximale plausible du statut de l'espèce grâce aux innovations et efforts de conservation durables à long terme (100 ans). Voir section IV.1 et Figure 1. 

en raison des propagules provenant d'autres unités spatiales. Une propagule est une entité vivante capable de se disperser et de produire un nouvel individu mature (p. ex. une spore, une graine, un fruit, un œuf, une larve, une partie d'un individu ou un individu entier). Les gamètes et le pollen ne sont pas considérés comme des propagules dans ce contexte (UICN, 2012b).

Unité spatiale

Subdivision de l'aire de répartition de l'espèce dans laquelle l'état de l'espèce est évalué comme étant Absent, Présent, Viable ou Fonctionnel. Voir «Délimitation des unités spatiales » à la section V, ci-dessous.

Population d'une unité spatiale

Ensemble des individus d'une espèce au sein d'une unité spatiale (cf. «population » dans UICN (2012a) et UICN (2019)). Également appelée «population dans une unité spatiale »

Catégorie de rétablissement de l'espèce
Mesure de la proximité de l'espèce par rapport à son Rétablissement complet, en fonction du Score de rétablissement de l'espèce et des seuils indiqués à la section IV.3.

\section{Score de rétablissement de l'espèce}

Mesure de la proximité de l'espèce par rapport à son Rétablissement complet, en fonction de la condition constatée, estimée, déduite ou supposée de l'espèce au moment de l'évaluation (voir Score vert "Actuel » à la Figure 1). II peut être tracé en fonction du temps, selon la condition à chaque étape temporelle, pour illustrer les progrès (ou non) de l'espèce vers un rétablissement complet.

État

Condition de l'espèce dans une unité spatiale, évaluée selon quatre catégories ordinales : Absent, Présent, Viable et Fonctionnel. Des pondérations sont attribuées à ces catégories (voir section V.3), lesquelles sont combinées à l'aide de l'Équation 1 ci-dessous pour obtenir le Score vert. L'état peut être déterminé pour les périodes passées, actuelles, futures à court terme et futures à long terme, ainsi que pour des scénarios alternatifs (avec ou sans conservation). La hiérarchie de ces états est imbriquée, de sorte que la population d'une unité spatiale dont l'état est évalué comme étant Viable est, par définition, également Présent ; de même, la population d'une unité spatiale dont l'état est évalué comme Fonctionnel est, par définition, également Viable et Présent. Par conséquent, un score Fonctionnel ne peut être appliqué qu'aux unités spatiales dont l'état est à la fois Viable et Fonctionnel (mais voir section V.3.d).

Sous-population

Par sous-populations, on entend des groupes distincts de la population, au plan géographique par exemple, entre lesquels les échanges démographiques ou génétiques sont limités (en règle générale une migration réussie d'un individu ou d'un gamète par an au plus) (UICN, 2012a ; voir Comité des normes et des pétitions de l'UICN (2019) pour plus de précisions).

Viable (état dans une unité spatiale)
L'état de la population d'une unité spatiale est dit «Viable » si l'espèce possède un faible risque de disparition dans l'unité spatiale, tel que déterminé par la catégorie de la Liste rouge de l'UICN au niveau régional. Voir Section V.3 pour plus de précisions. 


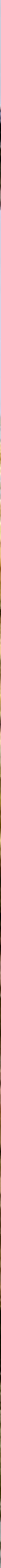




\section{Critères}

\section{Score vert}

L'état dans chaque unité spatiale est évalué selon quatre catégories ordinales : Absent, Présent, Viable et Fonctionnel (voir section V.3. pour plus de précisions). Un Score vert (G) pour l'espèce est obtenu en se basant sur les états dans toutes les unités spatiales, grâce à l'équation suivante (Équation 1) :

$$
G=\frac{\sum_{s} W_{s}}{W_{F} \times N} \times 100
$$

où s est chaque unité spatiale, $W_{S}$ est la pondération de l'état (Absent, Présent, Viable ou Fonctionnel) dans l'unité spatiale, $W_{F}$ est la pondération de l'état Fonctionnel, et $\mathrm{N}$ est le nombre d'unités spatiales. Le dénominateur est le score maximum possible atteint lorsque l'état de toutes les unités spatiales est évalué comme Fonctionnel. Ainsi, un Score vert est calculé en tant que pourcentage de Rétablissement complet. Pour les scores «Actuel » et « Actuel contrefactuel », le dénominateur est basé sur le nombre d'unités spatiales dans l'aire de répartition autochtone uniquement (sans inclure l'aire de répartition supplémentaire attendue).

Les différents Scores verts sont calculés en fonction des périodes actuelles, futures à court terme et futures à long terme, ainsi que des scénarios alternatifs, comme indiqué au Tableau 1. Les paramètres d'impact de la conservation (Héritage de la conservation, etc.) sont calculés en tant que différences entre deux Scores verts, comme cela est expliqué à la section V.

Tableau 1. Noms et descriptions des scénarios dans lesquels le Score vert peut être calculé. À noter : toutes les évaluations du Statut vert nécessitent au moins le scénario « Actuel »; pour évaluer l'impact de la conservation, au moins un autre scénario est nécessaire.

\begin{tabular}{|c|c|}
\hline Score vert & Scénario et horizon temporel \\
\hline Actuel & $\begin{array}{l}\text { Le Score vert au moment de l'évaluation (identique au Score de rétablissement de l'espèce à ce } \\
\text { moment-là). }\end{array}$ \\
\hline Actuel contrefactuel & $\begin{array}{l}\text { Ce que la valeur du Score vert aurait été aujourd'hui en l'absence d'actions de conservation } \\
\text { passées. Voir section V.4. }\end{array}$ \\
\hline Référence actuelle & $\begin{array}{l}\text { Valeur prévue du Score vert dans un avenir à court terme (10 ans), compte tenu des avantages } \\
\text { probables des actions de conservation actuellement en place ou très probablement en place d'ici } \\
\text { un an. Si le scénario « Référence actuelle » n'est pas précisé, il est supposé être identique au } \\
\text { scénario « Actuel ». Voir section V.5.c. }\end{array}$ \\
\hline Futur-avec-conservation & $\begin{array}{l}\text { Valeur prévue du Score vert dans un avenir à court terme (10 ans), compte tenu des avantages } \\
\text { probables des actions de conservation actuellement en place ou dont la mise en œuvre est plan- } \\
\text { ifiée durant cette période. Voir section V.5.a. }\end{array}$ \\
\hline Futur-sans-conservation & $\begin{array}{l}\text { Valeur prévue du Score vert dans un avenir à court terme (10 ans), en supposant que toute action } \\
\text { de conservation en cours s'arrête aujourd'hui et qu'aucune nouvelle action n'est mise en œuvre. } \\
\text { Voir section V.5.b. }\end{array}$ \\
\hline Potentiel à long terme & $\begin{array}{l}\text { Valeur prévue du Score vert à long terme (100 ans), compte tenu des innovations et actions de } \\
\text { conservation durables et efficaces. Voir section V.5.d. }\end{array}$ \\
\hline
\end{tabular}




\title{
2. Paramètres d'impact de la conservation
}

Pour toute espèce donnée, quatre paramètres d'impact de la conservation sont calculés en tant que différence entre deux Scores verts (voir Figure 1):

\author{
Héritage de la conservation $=$ Actuel - Actuel contrefactuel \\ (mesurant l'impact des actions de conservation menées à ce jour)
}

\section{Dépendance envers la conservation = Référence actuelle - Futur-sans-conservation} Gain par conservation = Futur-avec-conservation - Référence actuelle

(mesurant à la fois l'effet dans un avenir à court terme des actions de conservation en cours et planifiées)

Potentiel de rétablissement $\boldsymbol{=}$ Potentiel à long terme $\boldsymbol{-}$ Actuel

(mesurant l'effet futur à long terme des innovations et efforts de conservation plausibles)

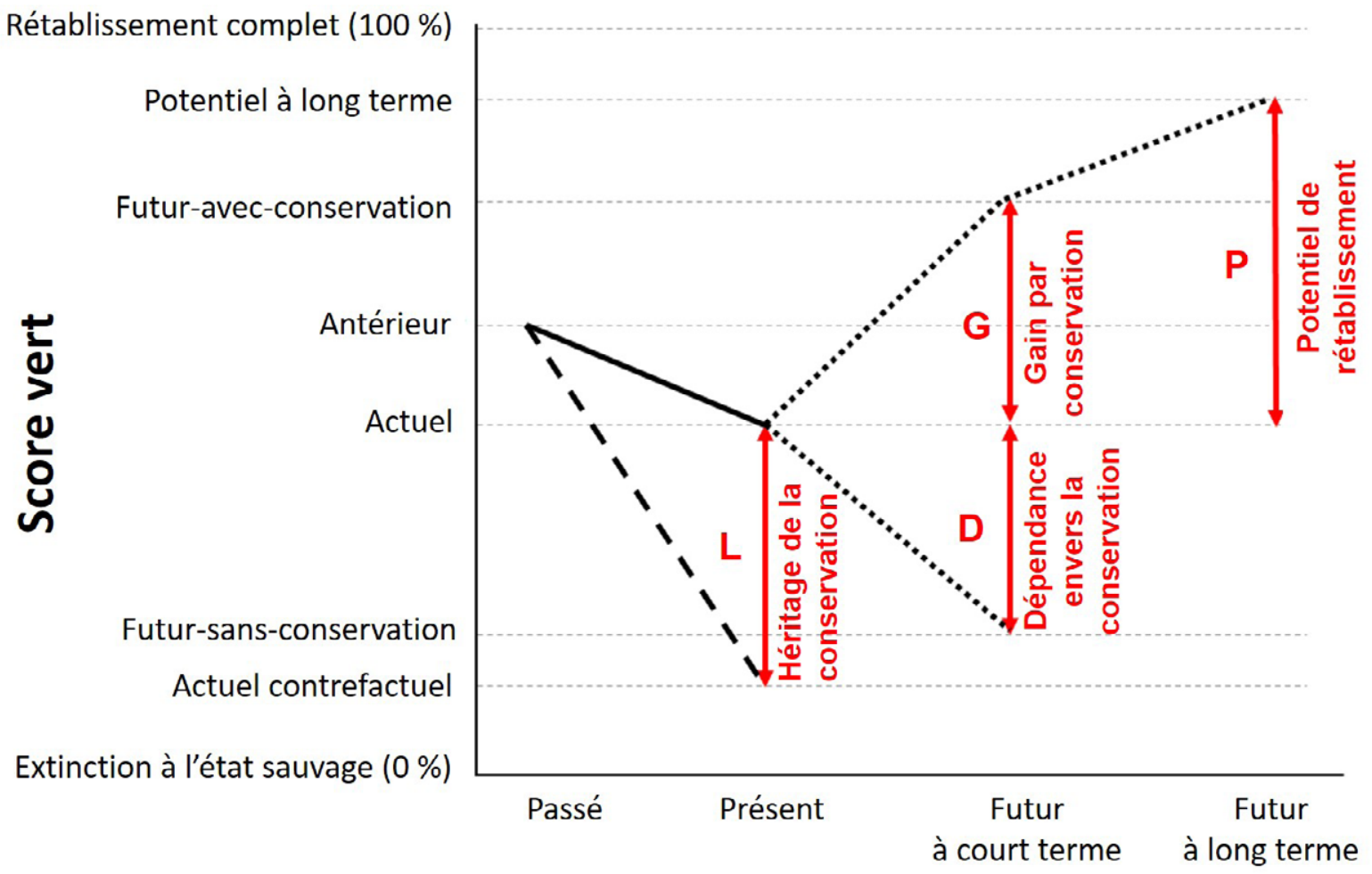

\section{Temps}

Figure 1. Représentation graphique des paramètres d'impact de la conservation en tant que différences de niveau de rétablissement de l'espèce (pourcentage de Rétablissement complet, mesuré en tant que Score vert ; équation 1). Ligne noire continue : changement constaté dans le Score vert de l'espèce. Ligne noire en pointillés longs : changement passé (contrefactuel) attendu en l'absence d'efforts de conservation passés. Lignes noires en pointillés courts : scénarios futurs de changements attendus avec et sans efforts de conservation actuels et futurs. Les flèches verticales représentent les paramètres d'impact de la conservation : Héritage de la conservation ( $L$ ) : avantages de la conservation actuelle et passée (actuel - actuel contrefactuel) ; Dépendance envers la conservation (D) : changement attendu dans un avenir à court terme en l'absence de conservation en cours (référence actuelle - futur-sans-conservation) ; Gain par conservation (G) : amélioration attendue dans un avenir à court terme avec la conservation en cours et planifiée (futur-avec-conservation - référence actuelle) ; Potentiel de rétablissement $(P)$ : amélioration possible avec une conservation à long terme (potentiel à long terme - actuel). Dans cet exemple, on suppose que la Référence actuelle (voir section V.5.c) a la même valeur que le score Actuel; voir le document Contexte et lignes directrices pour visualiser le même graphique avec une hypothèse alternative. 


\section{Catégories et seuils}

Dans la plupart des cas, il est préférable d'indiquer les valeurs numériques (en pourcentage) pour le Score de rétablissement de l'espèce et les quatre paramètres d'impact de la conservation, y compris leurs incertitudes (voir section V.8, ci-dessous). Toutefois, les résultats par catégorie peuvent être plus adaptés pour certaines utilisations et certains publics (voir section V.8). Par conséquent, chaque paramètre est divisé en catégories, afin de (i) fournir un contexte et permettre l'interprétation de la valeur en pourcentage du paramètre, et (ii) mettre en évidence les cas d'impact, de dépendance ou de potentiel de conservation exceptionnels.

Le Score de rétablissement de l'espèce (SRS) peut être indiqué soit numériquement (de 0 \% pour l'extinction à $100 \%$ pour le Rétablissement complet) soit par catégorie. Les Catégories de rétablissement de l'espèce sont basées sur la meilleure estimation, la valeur minimale et la valeur maximale du SRS (SRS ${ }_{\text {best }}, \mathrm{SRS}_{\min }$, $\mathrm{SRS}_{\text {max }}$, respectivement) et sur la meilleure estimation du paramètre Héritage de la conservation $\left(L_{\text {best }}\right)$, et elles sont attribuées selon les règles suivantes, lesquelles sont appliquées dans l'ordre indiqué jusqu'à ce que la condition donnée pour une catégorie soit remplie (Tableau 2).

Tableau 2. Catégories de rétablissement de l'espèce, basées sur la meilleure estimation, la valeur minimale et la valeur maximale du Score de rétablissement de l'espèce $\left(\mathrm{SRS}_{\text {best }}\right.$, $\mathrm{SRS}_{\min }$, $\mathrm{SRS}_{\max }$, respectivement) :

\begin{tabular}{|c|c|}
\hline Indéterminé & Si $\left(\mathrm{SRS}_{\max }-\mathrm{SRS}_{\min }\right)>40 \%$ \\
\hline Aucune diminution & Si $\left(\mathrm{SRS}_{\text {best }}=100 \%\right)$ et $\left(\mathrm{L}_{\text {best }}=0 \%\right)$ \\
\hline Rétablissement complet & Si $\mathrm{SRS}_{\text {best }}=100 \%$ \\
\hline Diminution légère & $\mathrm{Si} \mathrm{SRS}_{\text {best }}>80 \%$ \\
\hline Diminution modérée & Si SRS $_{\text {best }}>50 \%$ \\
\hline Diminution importante & Si SRS $_{\text {best }}>20 \%$ \\
\hline Diminution critique & Si $\mathrm{SRS}_{\text {best }}>0 \%$ \\
\hline Extinction à l'état sauvage & Si $\mathrm{SRS}_{\text {best }}=0 \%$ \\
\hline
\end{tabular}


La catégorie de chacun des quatre paramètres d'impact de la conservation est déterminée selon les conditions énumérées ci-dessous (Tableaux 3-6). Pour chaque paramètre, les règles sont appliquées dans l'ordre indiqué jusqu'à ce que la condition donnée pour une catégorie soit remplie. Dans ces conditions, la meilleure estimation, la valeur minimale et la valeur maximale des paramètres d'impact de la conservation, ainsi que les meilleures estimations des Scores verts ( $p$. ex. "Actuel » et «Actuel contrefactuel ») sont utilisées. Pour chaque paramètre, la catégorie «Élevé » peut être atteinte de l'une des trois manières suivantes : la valeur numérique est supérieure à $40 \%$; la valeur numérique est faible mais représente l'évitement de l'extinction ; ou la valeur numérique est faible mais importante par rapport à la meilleure estimation ou à l'estimation moyenne du score Actuel. Pour plus d'informations sur les seuils, voir le document Contexte et lignes directrices.

Tableau 3. Catégories d'Héritage de la conservation, basées sur la meilleure estimation, la valeur minimale et la valeur maximale du paramètre " Héritage de la conservation 》 $\left(L_{\text {best }}, L_{\text {min }}, L_{\max }\right.$, respectivement) :

\begin{tabular}{|ll|}
\hline Indéterminé & Si $L_{\text {min }}<0 \%$ et $L_{\text {max }}>40 \%$ \\
\hline Élevé & Si $L_{\text {best }}>40 \%$, ou \\
& $\begin{array}{l}L_{\text {best }}>0 \% \text { et Actuel contrefactuel=0 (c.-à-d. extinction évitée), ou } \\
L_{\text {best }}>1 / 2 \cdot \text { Actuel (c.-à-d. héritage important par rapport au score actuel) }\end{array}$ \\
\hline Moyen & Si $L_{\text {best }}>10 \%$ \\
\hline Faible & Si $L_{\text {best }}>0 \%$ \\
\hline Nul & Si $L_{\text {best }}=0 \%$ \\
\hline Négatif & Si $L_{\text {best }}<0 \%$ \\
\hline
\end{tabular}

À noter : Le caractère · est l'opérateur mathématique de la multiplication (identique à × )

Tableau 4. Catégories de Dépendance envers la conservation, basées sur la meilleure estimation, la valeur minimale et la valeur maximale du paramètre « Dépendance envers la conservation $\left(D_{\text {best }}\right.$, $D_{\min }, D_{\max }$, respectivement) :

\begin{tabular}{|ll|}
\hline Indéterminé & Si $D_{\min }<0 \%$ et $D_{\max }>40 \%$ \\
\hline Élevé & $\begin{array}{l}\text { Si } D_{\text {best }}>40 \% \text {, ou } \\
D_{\text {best }}>0 \% \text { et Futur-sans-conservation }=0 \text { (c.-à-d. sans conservation, l'espèce serait } \\
\text { éteinte), ou } \\
\end{array} \quad \begin{array}{l}D_{\text {best }}>1 / 2 \cdot \text { Actuel (ou Référence actuelle) (c.-à-d. dépendance importante par rapport } \\
\text { au score actuel) }\end{array}$ \\
\hline Moyen & Si $D_{\text {best }}>10 \%$ \\
\hline Faible & Si $D_{\text {best }}>0 \%$ \\
\hline Nul & Si $D_{\text {best }}=0 \%$ \\
\hline Négatif & Si $D_{\text {best }}<0 \%$ \\
\hline
\end{tabular}


Tableau 5. Catégories de Gain par conservation, basées sur la meilleure estimation, la valeur minimale et la valeur maximale du paramètre «Gain par conservation » $\left(G_{\text {best }}, G_{\min }, G_{\max }\right.$, respectivement) :

\begin{tabular}{|c|c|}
\hline Indéterminé & $\mathrm{Si} \mathrm{G}_{\min }<0 \%$ et $\mathrm{G}_{\max }>40 \%$ \\
\hline Élevé & $\begin{array}{l}\text { Si } G_{\text {best }}>40 \% \text {, ou } \\
G_{\text {best }}>0 \% \text { et Actuel = } 0 \text { (c.-à-d. sans conservation, l'espèce resterait EW), ou } \\
G_{\text {best }}>1 \cdot \text { Actuel (ou Référence actuelle) (c.-à-d. gain important par rapport au score } \\
\text { actuel) }\end{array}$ \\
\hline Moyen & Si $G_{\text {best }}>10 \%$ \\
\hline Faible & Si $G_{\text {best }}>0 \%$ \\
\hline Nul & Si $G_{\text {best }}=0 \%$ \\
\hline Négatif & $\mathrm{Si} \mathrm{G}_{\text {best }}<0 \%$ \\
\hline
\end{tabular}

Tableau 6. Catégories de Potentiel de rétablissement, basées sur la meilleure estimation, la valeur minimale et la valeur maximale du paramètre « Potentiel de rétablissement 》 $\left(P_{\text {best }}, P_{\min }, P_{\max }\right.$, respectivement) :

\begin{tabular}{|ll|}
\hline Indéterminé & Si $P_{\text {min }}<0 \%$ et $P_{\max }>40 \%$ \\
\hline Élevé & $\begin{array}{l}\text { Si } P_{\text {best }}>40 \% \text {, ou } \\
P_{\text {best }}>0 \% \text { et Actuel }=0 \text { (c. } \text {-à-d. sans conservation, l'espèce resterait EW), ou } \\
P_{\text {best }}>2 \cdot \text { Actuel (c.-à-d. rétablissement important par rapport au score actuel) }\end{array}$ \\
\hline Moyen & Si $P_{\text {best }}>10 \%$ \\
\hline Faible & Si $P_{\text {best }}>0 \%$ \\
\hline Nul & Si $P_{\text {best }}=0 \%$ \\
\hline Négatif & Si $P_{\text {best }}<0 \%$ \\
\hline
\end{tabular}




\section{Procédures}

Les procédures suivantes sont les principales composantes d'une évaluation du Statut vert. Ces procédures sont discutées en détail dans le document Contexte et lignes directrices pour le Statut vert des espèces de l'UICN, lequel évoluera au fil du temps pour intégrer des orientations sur l'utilisation des nouveaux types de données qui pourraient devenir disponibles, ainsi que les avancées concernant les méthodes d'analyse.

\section{Détermination de l'aire de répartition}

\section{a. Détermination de l'aire de répartition autochtone}

L'aire de répartition autochtone (voir définition) est fondée sur tous les sites d'occurrence connus, déduits et (spatialement) prévus, tant les sites actuels et historiques. Cela comprend les transferts de conservation conformes aux Lignes directrices du Comité des normes et des pétitions de l'UICN (2019), mais pas les individus errants. Pour que les objectifs de rétablissement soient ambitieux et réalisables, et pour éviter de modifier les références, l'aire de répartition autochtone devrait être basée sur la répartition à une date aussi précoce que possible, tout en reconnaissant que remonter trop loin dans le temps augmenterait les incertitudes en raison de la rareté des données. Idéalement, cette date devrait être fixée à " une époque antérieure à celle où les êtres humains étaient l'élément le plus important limitant la répartition des espèces » (Sanderson, 2019). Cependant, la variation entre les espèces en termes de disponibilité et de qualité des données signifie qu'un tel critère donnerait lieu à des évaluations risquant de ne pas être comparables. Par conséquent, les espèces de la même région géographique et soumises à des menaces similaires devraient avoir des dates de référence similaires pour déterminer l'aire de répartition autochtone (Stephenson et al., 2019). En se basant sur l'analyse des tendances des données historiques concernant la couverture des habitats et les populations humaines (d'après Klein Goldewijk et al., 2011), une date de référence par défaut de 1750 de notre ère convient dans la plupart des régions et est conforme à la définition du début de l'ère industrielle selon le GIEC. Cette date de référence peut être modifiée (voir le document Contexte et lignes directrices), mais ne doit pas être antérieure à 1500 de notre ère ou postérieure à 1950.

\section{b. Détermination de l'aire de répartition supplémentaire attendue}

L'aire de répartition supplémentaire attendue comprend uniquement les zones qui : (i) ne font pas partie de l'aire de répartition autochtone ; et (ii) sont actuellement adaptées ou fortement susceptibles de le devenir au cours des 100 prochaines années ; et (iii) sont fortement susceptibles d'être occupées par l'espèce au cours des 100 prochaines années, avec ou sans assistance humaine (à condition que celles bénéficiant d'une assistance humaine soient conformes aux Lignes directrices du Comité des normes et des pétitions de l'UICN (2019)). Cette probabilité peut être évaluée sur la base d'une modélisation (p. ex., sur les changements induits par le climat), des transferts planifiés ou de l'observation empirique des changements d'aire de répartition.

En raison des horizons temporels généralement utilisés pour faire une projection des changements d'aire de répartition dus au changement climatique, l'aire de répartition supplémentaire attendue ne serait normalement utilisée que pour le futur à long terme (c'est-à-dire pour calculer le Potentiel à long terme). À moins que certaines unités spatiales situées dans l'aire de répartition supplémentaire attendue ne deviennent occupées ou ne fassent l'objet d'efforts de conservation au cours des 10 prochaines années, les scénarios d'avenir à court terme (pour le calcul de la Dépendance envers la conservation et du Gain par conservation) n'ont pas besoin de tenir compte de l'aire de répartition supplémentaire attendue.

\section{c. Individus gérés et introduits}

Pour l'aire de répartition autochtone comme pour l'aire de répartition supplémentaire attendue, seuls les individus " sauvages » de l'espèce devraient être pris en compte pour déterminer l'état dans chaque unité spatiale. Pour une définition du terme " sauvage », voir la dernière version des Lignes directrices pour la Liste rouge (Comité des normes et des pétitions de l'UICN, 2019), section 2.1.4.

Pour l'aire de répartition autochtone et l'aire de répartition supplémentaire attendue, les sous-populations sauvages résultant d'introductions en dehors de l'aire de répartition autochtone de l'espèce devraient être prises en compte pour déterminer l'état, à condition que certaines conditions soient remplies (voir la dernière version des Lignes directrices pour la Liste rouge (UICN, 2019), section 2.1.3). 


\section{Délimitation des unités spatiales}

Les unités spatiales sont utilisées pour intégrer la représentation, l'une des trois exigences sur lesquelles repose la définition d'une espèce entièrement rétablie (voir II.3). Une espèce entièrement rétablie se présente sous la forme d'un ensemble de populations fonctionnelles au sein d'un ensemble représentatif d'écosystèmes et de communautés à l'intérieur de son aire de répartition. Une façon pratique d'évaluer cette condition consiste à déterminer l'état de l'espèce dans chacune des unités spatiales qui composent son aire de répartition, en les délimitant de manière à représenter la variation des conditions ou paramètres écologiques dans l'aire de répartition de l'espèce. Les unités spatiales doivent être choisies avec soin car le Score vert est sensible au nombre d'unités. Étant donné que les unités spatiales ont la même valeur dans le calcul des Scores verts, elles doivent être définies de manière à représenter des zones d'importance similaire pour la conservation de l'espèce, tant en ce qui concerne la représentation globale que les actions de conservation, sur toute l'étendue de l'aire de répartition autochtone de l'espèce et de son aire de répartition supplémentaire attendue.

Les unités spatiales peuvent être délimitées en fonction de la sous-population, des caractéristiques écologiques et géographiques, de l'emplacement ou d'une combinaison de ces éléments. Les subdivisions spécifiques aux espèces fondées sur la biologie des espèces, telles que les sous-populations (définies dans les Lignes directrices du Comité des normes et des pétitions de l'UICN (2019)), sont préférables. Les sous-espèces, les stocks, les unités génétiques, les voies de migration, les unités significatives sur le plan de l'évolution et les segments de population distincts sont tous conceptuellement liés à la définition d'une sous-population selon I'UICN. Bien qu'elles ne soient pas spécifiques aux espèces, les divisions basées sur les écorégions, les types d'habitats ou les types d'écosystèmes peuvent également être utilisées pour définir les unités spatiales, car elles sont définies en fonction de critères écologiques et rendent ainsi compte des différents contextes écologiques dans lesquels une espèce existe ou a existé. Les caractéristiques géographiques (par exemple, les bassins versants, les îles, les lacs, les chaînes montagneuses) peuvent être des substituts aux sous-populations. La fragmentation récente de l'espèce en " sous-populations » en raison de l'activité humaine n'est pas une base adaptée pour délimiter les unités spatiales, si ces « sous-populations » étaient historiquement liées. Enfin, les zones définies en raison de leur vulnérabilité à une menace spécifique ("Localité » dans UICN (2019)) peuvent être utilisées pour définir des unités spatiales, en partant du principe que le statut de l'espèce sera similaire dans toute une zone menacée de manière similaire.

De nombreuses espèces ayant une aire de répartition restreinte peuvent être évaluées sur la base d'une seule unité spatiale, ou de deux unités spatiales (par exemple, l'une pour l'aire de répartition existante et l'autre pour l'aire de répartition disparue). Cela peut aussi être le cas pour une espèce qui a toujours existé dans un type d'écosystème très spécifique ou pour une espèce dont la fonction est similaire dans les différents contextes écologiques dans lesquels elle existe. Pour d'autres espèces, trois unités spatiales ou plus peuvent être nécessaires pour représenter la variété des conditions et contextes écologiques dans lesquels l'espèce est ou a été présente.

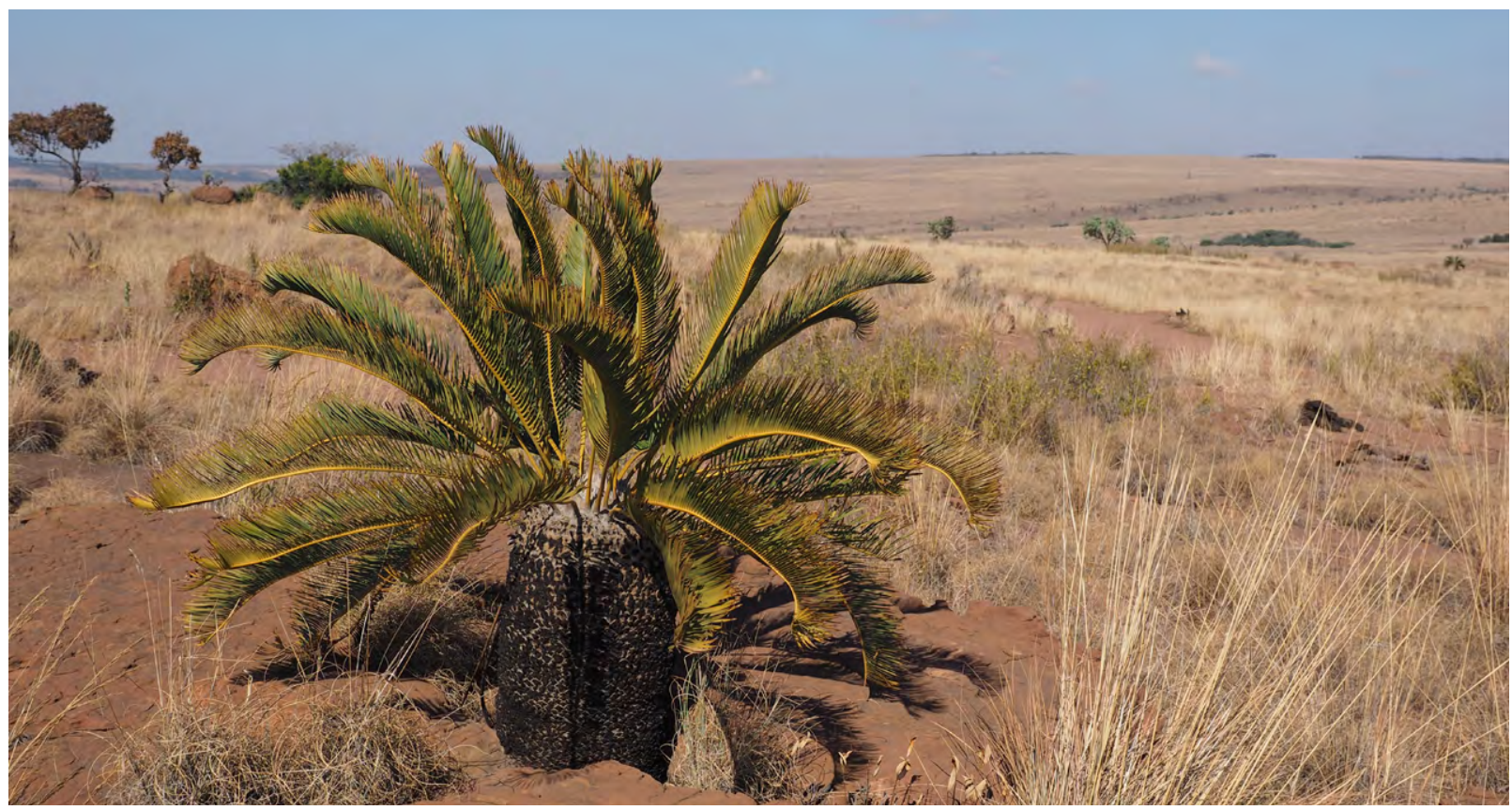

Cycas laineux (Encephalartos lanatus) @ Michael Calonje 


\section{3. Évaluation de l'état dans une unité spatiale}

L'état dans chaque unité spatiale est évalué de manière séquentielle dans l'ordre suivant : Absent, Présent, Viable, Fonctionnel. La définition des termes Absent, Présent et Viable nécessite l'utilisation des catégories de la Liste rouge ; ainsi, les évaluateurs devraient consulter la dernière version des documents de la Liste rouge (UICN, 2012a ; UICN, 2012b ; Comité des normes et des pétitions de l'UICN, 2019). Les évaluateurs doivent documenter l'état le plus probable dans chaque unité spatiale ainsi que les états minimum et maximum plausibles, afin de traduire le niveau d'incertitude. Les lignes directrices pour la Liste rouge régionale doivent être utilisées à moins qu'il n'y ait qu'une seule Unité spatiale pour l'ensemble de l'espèce.

\section{a. Évaluation de l'Absence}

L'espèce est considérée Absente dans une unité spatiale si la catégorie de la Liste rouge dont elle relève dans l'unité spatiale concernée est Éteint (EX), Éteint à l'état sauvage (EW), Éteint au niveau régional (RE), En danger critique (Peut-être éteint) (CR(PE)) ou En danger critique (Peut-être éteint à l'état sauvage) (CR(PEW)). Concernant les méthodes permettant de faire la distinction entre la catégorie «Éteint » et la mention " Peut-être éteint », voir la dernière version des Lignes directrices pour la Liste rouge. La pondération pour l'état « Absent » dans l'Équation 1 est 0.

\section{b. Évaluation de la Présence}

L'espèce est considérée Présente dans une unité spatiale si elle s'y trouve (c.-à-d. elle n'est pas Absente), et si soit (i) elle relève, dans l'unité spatiale, d'une catégorie menacée selon la Liste rouge au niveau régional, à savoir Vulnérable (VU), En danger (EN) ou En danger critique (CR), mais pas CR(PE) ou CR(PEW), soit (ii) la catégorie est Quasi menacé (NT) et la population de l'unité spatiale est en « déclin continu » en termes d'effectifs, selon la définition du Comité des normes et des pétitions de I'UICN (2019). Dans les unités spatiales dépourvues d'individus matures, la présence d'individus immatures (y compris les banques de graines) suffit pour que l'état dans l'unité spatiale soit évalué comme étant « Présent ». De même, l'état dans les unités spatiales comportant une « dette d'extinction » (c.-à-d. qu'un recrutement insuffisant provoquera à terme une extinction locale) est évalué comme étant «Présent ", pas " Absent ». Une évaluation de la Liste rouge au niveau régional (UICN, 2012b) nécessite des dispositions pour la possibilité d'un effet de sauvetage suite à une immigration depuis d'autres unités spatiales, et peut entraîner un déclassement de la catégorie de menace, par exemple de VU à NT (voir UICN (2012b)). L'état dans l'unité spatiale doit être basé sur la catégorie après tout ajustement applicable à la catégorie de menace.

La pondération par défaut pour l'état « Présent » dans l'Équation 1 est 3. Toutefois, à la place de cette valeur par défaut, les évaluateurs peuvent utiliser la pondération correspondant spécifiquement à la catégorie de la Liste rouge au niveau régional pour la population de l'unité spatiale : 1,5 pour CR, 2,5 pour EN, 3,5 pour VU ou 4,5 pour NT avec un déclin continu (voir le document Contexte et lignes directrices qui explique ces pondérations affinées). Si la catégorie de la Liste rouge est DD, il faut utiliser les pondérations correspondant à l'état « Absent » (zéro) pour la valeur minimum et à l'état « Fonctionnel » (9) pour la valeur maximum.

\section{c. Évaluation de la Viabilité}

L'espèce est considérée Viable dans une unité spatiale si une évaluation de la Liste rouge au niveau régional (UICN, 2012b) de l'espèce au sein de cette unité spatiale aboutit à la désignation « Préoccupation mineure » (LC), ou bien la désignation est «Quasi menacé » (NT) et la population de l'unité spatiale n'est pas en « déclin continu » en termes d'effectifs, selon la définition du Comité des normes et des pétitions de l'UICN (2019). Une évaluation de la Liste rouge au niveau régional (UICN, 2012b) nécessite des dispositions pour la possibilité d'un effet de sauvetage suite à une immigration depuis d'autres unités spatiales, et peut entraîner un déclassement de la catégorie de menace, par exemple de NT à LC (voir UICN (2012b)). L'état dans l'unité spatiale doit être basé sur la catégorie après tout ajustement applicable à la catégorie de menace.

La pondération par défaut pour l'état « Viable » dans l'Équation 1 est 6 . Toutefois, à la place de cette valeur par défaut, les évaluateurs peuvent utiliser la pondération correspondant spécifiquement à la catégorie de la Liste rouge au niveau régional pour la population de l'unité spatiale : 5,5 pour NT sans déclin continu, ou 6,5 pour LC. Si la catégorie est DD, il faut utiliser les pondérations correspondant à l'état «Absent » pour la valeur minimum et à l'état « Fonctionnel » pour la valeur maximum. 


\section{d. Évaluation de la Fonctionnalité}

Tout comme la viabilité, la fonctionnalité est évaluée au sein de chaque unité spatiale. II faut pour cela évaluer chaque unité spatiale par rapport aux fonctions qui constituent les rôles les plus importants de l'espèce. Bien que ces fonctions puissent ne pas être faciles à déterminer, l'intégration de la fonctionnalité chaque fois que cela est possible est un élément essentiel d'une vision ambitieuse de la conservation. Cependant, la prise en compte de la fonctionnalité dans le contexte du rétablissement des espèces ne doit pas être interprétée à tort comme une priorité donnée à la conservation des espèces en fonction de leur importance fonctionnelle.

Contrairement aux autres états (Absent, Présent et Viable), l'état «Fonctionnel » n'est pas basé sur les critères de la Liste rouge (mais voir la section sur le lien entre la Viabilité et la Fonctionnalité dans le document Contexte et lignes directrices). La fonctionnalité peut être déterminée directement, en considérant les interactions de l'espèce et ses contributions aux processus écosystémiques, ou indirectement, en recherchant les symptômes d'une fonctionnalité réduite, par analogie avec l'approche de la Liste rouge qui consiste à identifier les symptômes d'une viabilité réduite (Akçakaya et al., 2020). Lorsqu'une fonction ne peut pas être identifiée pour une espèce, un certain nombre de substituts peuvent être utilisés pour évaluer la fonctionnalité, notamment la densité de population ou la structure d'âge dans les zones à faible impact humain ou à un niveau de référence historique.

Bien qu'en principe, une population puisse contribuer à un processus écosystémique particulier même lorsqu'elle court un risque élevé de disparition, aux fins du processus d'évaluation du Statut vert, le score Fonctionnel n'est appliqué qu'aux unités spatiales qui sont également Viables. Une exception est le rare cas d'une unité spatiale ayant une population naturellement petite (<1000 individus matures) qui remplit ses fonctions écologiques aux niveaux de référence, mais qui ne répondrait pas aux critères de l'état Viable car elle serait classée comme VU selon le critère D de la Liste Rouge. Si une telle population d'une unité spatiale n'est pas en déclin, ne fait pas l'objet d'une menace spécifique et ne répond pas aux autres critères de la catégorie VU, son état peut être considéré comme Fonctionnel.

Si les unités spatiales sont basées sur des sous-populations, alors l'état dans une unité spatiale est évalué comme étant Fonctionnel si la sous-population est Fonctionnelle, selon la définition ci-dessus. Si les unités spatiales ne sont pas basées sur des sous-populations et qu'il existe plusieurs sous-populations par unité spatiale, l'état dans une unité spatiale est considéré comme Fonctionnel si plus de la moitié des sous-populations de cette unité spatiale sont fonctionnelles (mais si l'on utilise des pondérations affinées, le seuil est différent ; voir ci-dessous).

La pondération par défaut pour l'état «Fonctionnel » dans l'Équation 1 est 9. Toutefois, à la place de cette valeur par défaut, les évaluateurs peuvent utiliser la pondération correspondant à la proportion des sous-populations dont l'état a été évalué comme étant Fonctionnel au sein de l'unité spatiale : 8 si < 40 \%, 9 si 40-70 \%, 10 si > $70 \%$.

\section{4. Élaboration du scénario Actuel contrefactuel}

Le scénario «Actuel contrefactuel » est un présent alternatif, envisagé afin de déterminer ce que le Score Vert «Actuel » aurait été si aucune action de conservation n'avait été menée. L'élaboration du scénario contrefactuel nécessite de déterminer comment la totalité des actions de conservation depuis 1950 a affecté la « trajectoire » de la population de l'espèce au cours de cette période, afin de pouvoir estimer ce que l'état aurait été aujourd'hui dans chacune des unités spatiales si ces actions de conservation n'avaient pas eu lieu. La réflexion contrefactuelle est un outil courant dans le domaine de la conservation (voir le document Contexte et lignes directrices pour obtenir des précisions et des instructions).

Lors de l'élaboration du scénario contrefactuel, les types d'informations à prendre en compte comprennent les effectifs et les tendances de la population, les changements en termes de répartition et de disponibilité de l'habitat, la gravité, la portée et l'intensité des menaces, et toutes les actions de conservation qui ont été mises en œuvre depuis le début des principales actions de conservation. Les actions de conservation qui étaient en place en 1950 et toutes celles qui ont suivi doivent être prises en compte. Les évaluateurs doivent indiquer l'année de début des actions de conservation passées qui ont été prises en compte dans leur évaluation. En outre, les évaluateurs doivent tenir compte des informations sur l'efficacité de chaque type d'action de conservation dans le contexte plus large de l'évolution des pressions et des opportunités de conservation pour les espèces de l'unité spatiale.

Si aucune action de conservation passée n'a été menée, le scénario actuel contrefactuel est le même que le scénario actuel. 


\section{5. Élaboration des scénarios futurs}

Un scénario futur projette l'état futur de chaque unité spatiale selon différentes hypothèses, dans le but de calculer les paramètres Gain par conservation, Dépendance envers la conservation et Potentiel de rétablissement. Les types d'informations à prendre en compte pour l'élaboration des scénarios futurs sont similaires à ceux nécessaires pour le scénario «Actuel contrefactuel » discuté plus haut. L'évaluateur doit prendre en considération à la fois les menaces actuelles et les menaces futures plausibles. Les menaces futures doivent être basées sur des preuves spécifiques (telles que des plans de développement, des projections socioéconomiques, etc.) et ne doivent pas être spéculatives.

\section{a. Futur-avec-conservation}

Pour le scénario « Futur-avec-conservation » (pour évaluer le Gain par conservation), les évaluateurs doivent en outre prendre en compte les effets probables de toutes les interventions de conservation actuellement en place ou planifiées pendant la période d'évaluation de 10 ans. Cependant, les évaluateurs ne doivent pas prendre en compte les actions de conservation envisagées mais non planifiées (par exemple, si aucun objectif clair, aucune estimation des coûts ou aucun calendrier n'est spécifié(e)) ni les actions de conservation planifiées mais dont le démarrage n'est pas prévu au cours de la période de 10 ans. Pour les actions planifiées, les évaluateurs doivent faire des hypothèses réalistes sur (i) la probabilité que l'action soit mise en œuvre, et (ii) la probabilité que les actions de conservation aient un effet positif sur la population d'une espèce dans une unité spatiale donnée. Pour les actions en place, les évaluateurs doivent considérer la probabilité (ii). Les avantages probables attendus grâce à ces mesures de conservation doivent être ajustés en fonction de ces probabilités.

\section{b. Futur-sans-conservation}

Pour le scénario « Futur-sans-conservation » (pour évaluer la Dépendance envers la conservation), les évaluateurs doivent prendre en compte les effets probables de toutes les interventions de conservation actuellement en place ou planifiées, puis exclure de leurs projections les effets correspondants, de la même manière que l'on retire les effets des actions de conservation passées dans un scénario contrefactuel.

\section{c. Référence actuelle}

Les deux scénarios futurs décrits dans les sections précédentes ( $5 a$ et $5 b$ ) sont comparés au scénario « Référence actuelle » afin de calculer les paramètres Gain par conservation et Dépendance envers la conservation. La Référence actuelle évalue l'état probable de la population de l'unité spatiale après 10 ans, en se basant uniquement sur les actions de conservation en cours (y compris les actions dont la mise en œuvre est hautement probable dans un délai d'un an, avec le financement et les autorisations en place), en tenant compte des menaces actuelles et des menaces futures plausibles. Les menaces futures doivent être basées sur des preuves spécifiques (telles que des plans de développement, des projections socioéconomiques, etc.) et ne doivent pas être spéculatives. Le scénario « Référence actuelle » tient compte du potentiel de déclin ou de rétablissement continu de la population d'une unité spatiale, indépendamment des actions de conservation planifiées ou de leur abandon. Si les évaluateurs ne souhaitent pas calculer un scénario "Référence actuelle », celui-ci est considéré par défaut comme étant le statut « Actuel ».

\section{d. Potentiel à long terme}

Pour le scénario « Potentiel à long terme » (pour évaluer le Potentiel de rétablissement), les évaluateurs doivent envisager les innovations et efforts de conservation plausibles qui pourraient avoir lieu au cours des 100 prochaines années. Cela comprend les actions qui pourraient être menées pour éliminer les menaces, ainsi que les possibilités de restauration de l'habitat et de connectivité accrue. Ce scénario doit être réaliste, compte tenu des limites biologiques de l'espèce (par exemple, la durée générationnelle et le taux maximal d'augmentation de la population) et de son habitat (par exemple, les taux de régénération). II doit également être réaliste en termes de facteurs sociaux, culturels et économiques (par exemple, les tendances prévues en matière d'urbanisation), mais le potentiel à long terme ne doit pas être limité par les contraintes politiques ou budgétaires actuelles. Ce scénario est comparé au statut "Actuel » (et non à la « Référence actuelle ") afin d'évaluer le Potentiel de rétablissement. Le Potentiel à long terme n'est pas censé être une prédiction exacte, car il serait impossible de prévoir avec précision tous les changements naturels, sociaux, économiques et technologiques qui se produiront au cours des 100 prochaines années. II s'agit plutôt d'une prévision raisonnable de l'ampleur du rétablissement de l'espèce, compte tenu des connaissances actuelles. 


\section{Intégration des incertitudes}

L'incertitude concernant l'état de l'espèce (Absent, Présent, Viable, Fonctionnel) dans chaque unité spatiale et pour chaque scénario doit être explicitée en précisant : (i) l'état plausible minimal ; (ii) l'état plausible maximal et (iii) l'état le plus probable (le meilleur). Ces incertitudes sont utilisées pour calculer les valeurs minimales et maximales des quatre paramètres d'impact de la conservation (voir le document Contexte et lignes directrices, et Akçakaya et al. (2018) Annexe S1).

\section{Documentation des évaluations}

Afin de garantir que les évaluations sont pleinement justifiées et de permettre l'analyse des données d'évaluation, un ensemble d'informations justificatives minimales est requis. Ces données facilitent la transparence et la reproductibilité, et permettent aux utilisateurs de rechercher et de trouver facilement des informations sur le site Web. Le document Contexte et lignes directrices pour le Statut vert des espèces de I'UICN fournit des orientations sur les éléments suivants : (i) informations justificatives requises pour toutes les évaluations du Statut vert ; (ii) informations justificatives requises dans des conditions spécifiques ; et (iii) informations justificatives recommandées. Il est à noter que les normes de documentation seront mises à jour de temps à autre.

\section{Communication des résultats des évaluations}

La manière de présenter les résultats d'une évaluation dépend du public qui les utilisera et de la façon dont il les utilisera. Pour certains publics, seuls un ou deux des quatre paramètres d'impact de la conservation peuvent être pertinents ; pour d'autres, les quatre peuvent être présentés. Pour la plupart des analyses impliquant plusieurs espèces, et pour la plupart des objectifs de recherche, les valeurs numériques (pourcentages) des quatre paramètres d'impact de la conservation, et leurs limites d'incertitude, devraient être utilisées. À d'autres fins, les résultats peuvent être communiqués en conjuguant ces valeurs numériques et les catégories, conformément aux catégories et aux seuils spécifiés plus haut (voir « Catégories et seuils ", IV.3).

\section{9. Évaluations régionales (dont les évaluations nationales)}

Les évaluations du Statut vert à des échelles spatiales régionales sont possibles, mais nécessitent une prise en compte attentive des aspects « aire de répartition autochtone et aire de répartition supplémentaire attendue » et " parties de l'aire de répartition » dans la définition du terme « Rétablissement complet ». II est fortement recommandé que les évaluations régionales (y compris les évaluations nationales) ne soient effectuées qu'à l'issue des deux premières étapes des évaluations mondiales : détermination de l'aire de répartition autochtone et de l'aire de répartition supplémentaire attendue, et délimitation des unités spatiales.

Dans la mesure du possible, l'aire de répartition de l'espèce considérée dans une évaluation régionale doit inclure intégralement une ou plusieurs unités spatiales figurant dans l'évaluation mondiale. En d'autres termes, les évaluations régionales ou nationales doivent éviter de diviser une unité spatiale déterminée et délimitée aux fins d'une évaluation mondiale. En incluant des unités spatiales entières (visées par l'évaluation mondiale) dans les évaluations régionales, il sera possible de combiner les résultats de deux ou plusieurs évaluations régionales et donc de faciliter le flux d'informations entre les évaluations régionales et mondiales. 


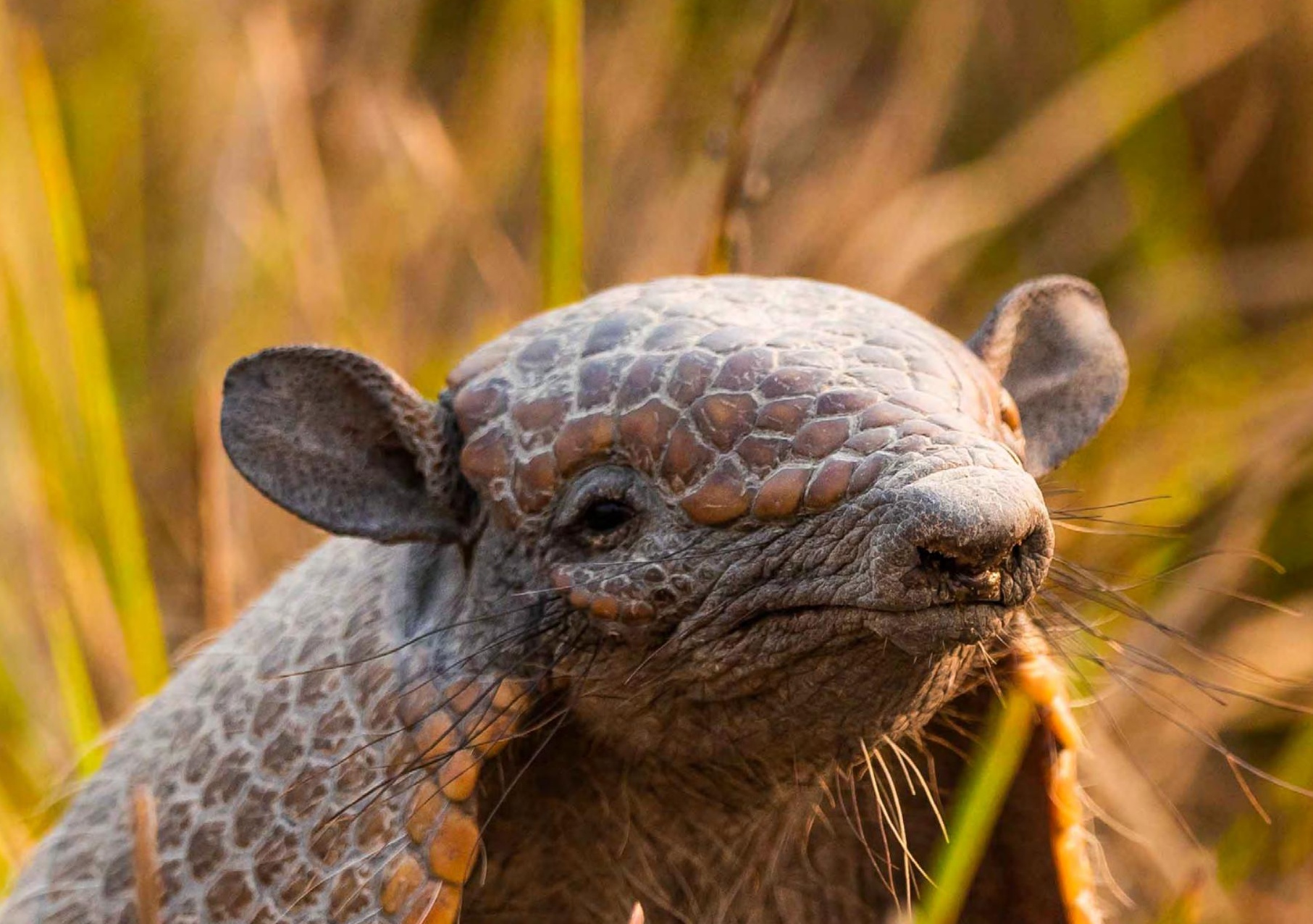

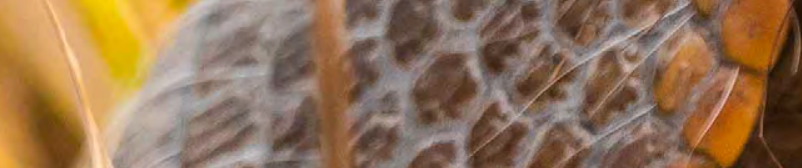

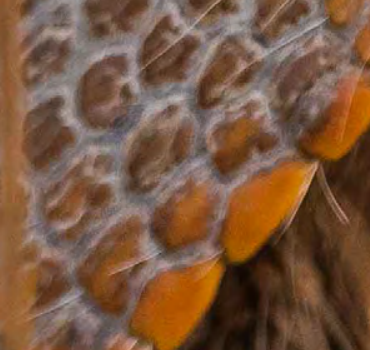

(1)

136
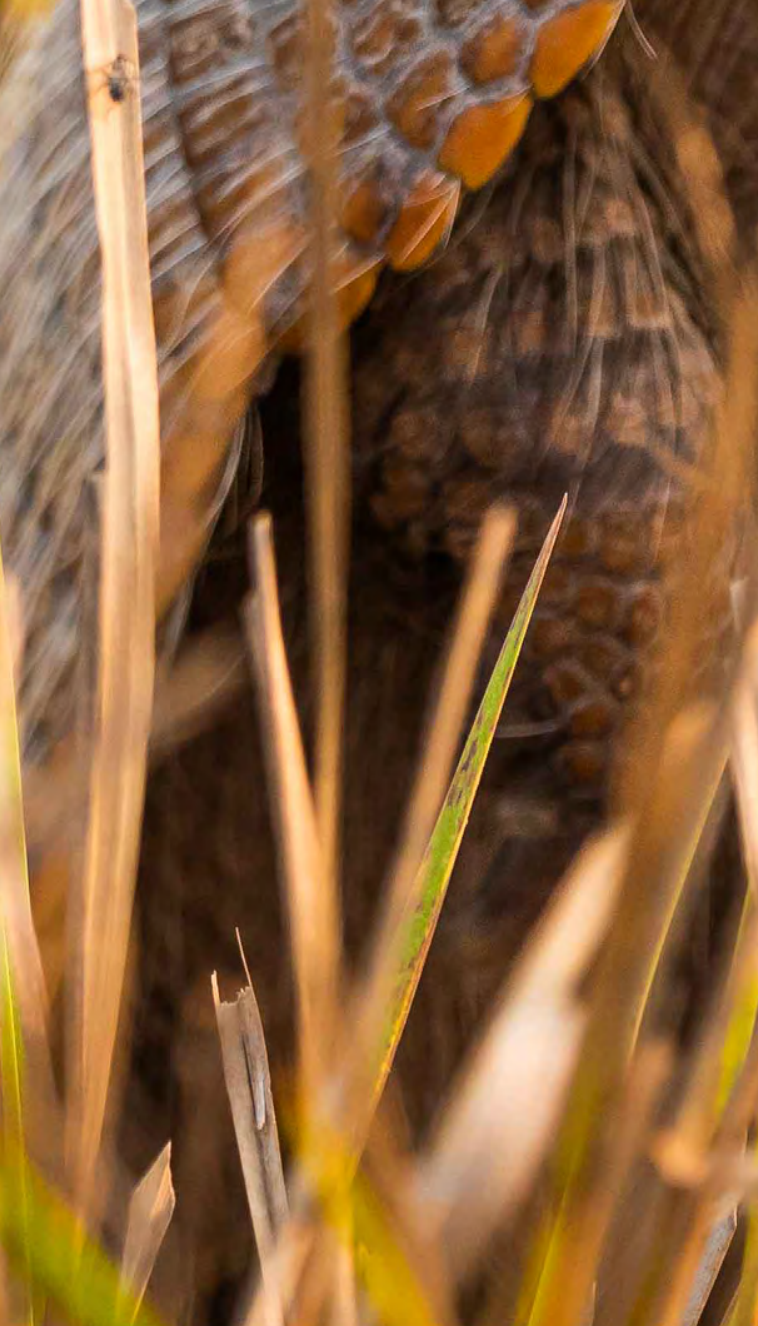

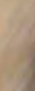




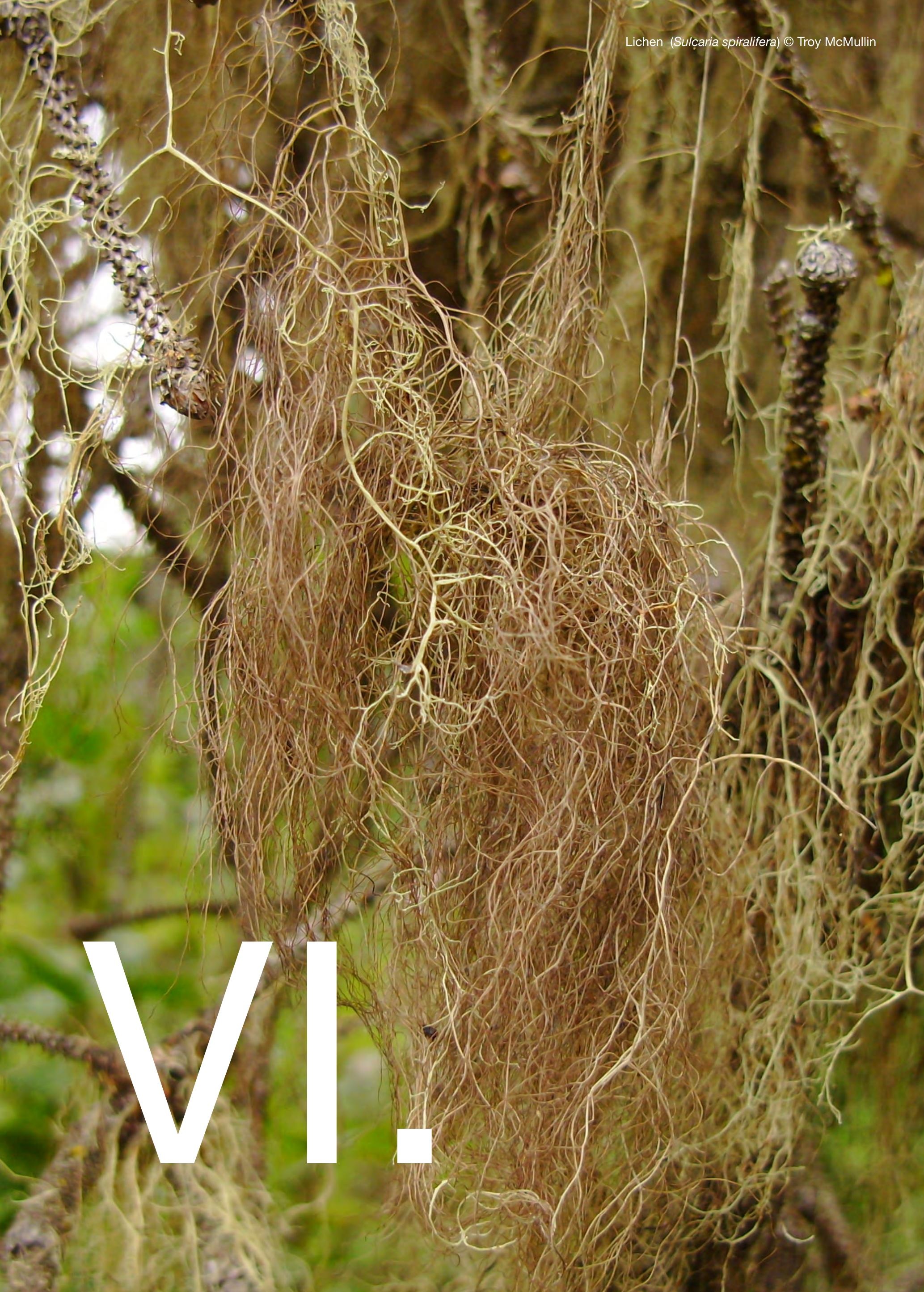




\section{Références}

Akçakaya, H.R., Bennett, E.L., Brooks, T.M., Grace, M.K., Heath, A., Hedges, S., Hilton-Taylor, C., Hoffmann, M., Keith, D.A., Long, B., Mallon, D.P., Meijaard, E., Milner-Gulland, E.J., Rodrigues, A.S.L., Rodriguez, J.P., Stephenson, P.J., Stuart, S.N. and Young, R.P. (2018). 'Quantifying species recovery and conservation success to develop an IUCN Green List of Species'. Conservation Biology 32:1128-1138. Available at: https://doi.org/10.1111/ cobi.13112 (Accessed: 10 September 2020).

Akçakaya, H.R., Rodrigues, A.S.L., Keith, D.A., Milner-Gulland, E.J., Sanderson, E.W., Hedges, S., Mallon, D.P., Grace, M.K., Long, B., Meijaard, E. and Stephenson, P.J. (2020). 'Assessing ecological function in the context of species recovery'. Conservation Biology 34:561-571. Available at: https://doi.org/10.1111/cobi.13425 (Accessed: 10 September 2020).

Dudley, N. and Timmins, H.L. (eds.) (2021). A survey of user attitudes towards the proposed IUCN Green Status of Species. Gland, Switzerland: IUCN. https://doi.org/10.2305/IUCN.CH.2021.03.en

International Union for Conservation of Nature (IUCN) Standards and Petitions Committee (2019). Guidelines for Using the IUCN Red List Categories and Criteria. Version 14. Prepared by the Standards and Petitions Committee. Downloadable from http://www.iucnredlist.org/documents/RedListGuidelines.pdf

International Union for Conservation of Nature (IUCN) and World Commission on Protected Areas (WCPA) (2017). IUCN Green List of Protected and Conserved Areas: Standard, Version 1.1. Gland, Switzerland: IUCN.

Klein Goldewijk, K., Beusen, A., van Drecht, G. and de Vos, M. (2011). 'The HYDE 3.1 spatially explicit database of human-induced global land-use change over the past 12,000 years'. Global Ecology and Biogeography 20:73-86. Available at: https://doi.org/10.1111/j.1466-8238.2010.00587.x (Accessed: 10 September 2020).

Sanderson, E.W. (2019). 'A full and authentic reckoning of species' ranges for conservation: response to Akçakaya et al. 2018'. Conservation Biology 33:1208-1210. Available at: http://doi.org/10.1111/cobi.13399 (Accessed: 10 September 2020).

Stephenson, P.J., Grace, M.K., Akçakaya, H.R., Rodrigues, A.S.L., Long, B., Mallon, D.P., Meijaard, E., Rodriguez, J.P., Young, R.P., Brooks, T.M. and Hilton-Taylor, C. (2019). 'Defining the indigenous ranges of species to account for geographic and taxonomic variation in the history of human impacts: reply to Sanderson 2019'. Conservation Biology 33:1211-1213. Available at: http://doi.org/10.1111/cobi.13400 (Accessed: 10 September 2020).

Union internationale pour la conservation de la nature : (UICN) (2012a). Catégories et Critères de la Liste rouge de I'UICN : Version 3.1. Deuxième édition. Gland, Suisse et Cambridge, Royaume-Uni : UICN. https://portals.iucn. org/library/node/10317

Union internationale pour la conservation de la nature : (UICN) (2012b). Lignes directrices pour l'application des Critères de la Liste rouge de l'UICN aux niveaux régional et national : Version 4.0. Gland, Suisse et Cambridge, Royaume-Uni : UICN. https://portals.iucn.org/library/node/10337

Union internationale pour la conservation de la nature : (UICN) (2013). Lignes directrices de l'UICN sur les reintroductions et les autres transferts aux fins de la sauvegarde. Gland, Suisse : UICN CSE. https://portals.iucn.org/ library/node/45128 




\section{UICN}

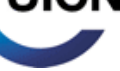

UNION INTERNATIONALE POUR LA CONSERVATION DE LA NATURE

SIÈGE MONDIAL

Rue Mauverney 28

1196 Gland, Suisse

mail@iucn.org

Tél. +41229990000

Fax +41229990002

www.iucn.org/fr

www.iucn.org/resources/publications

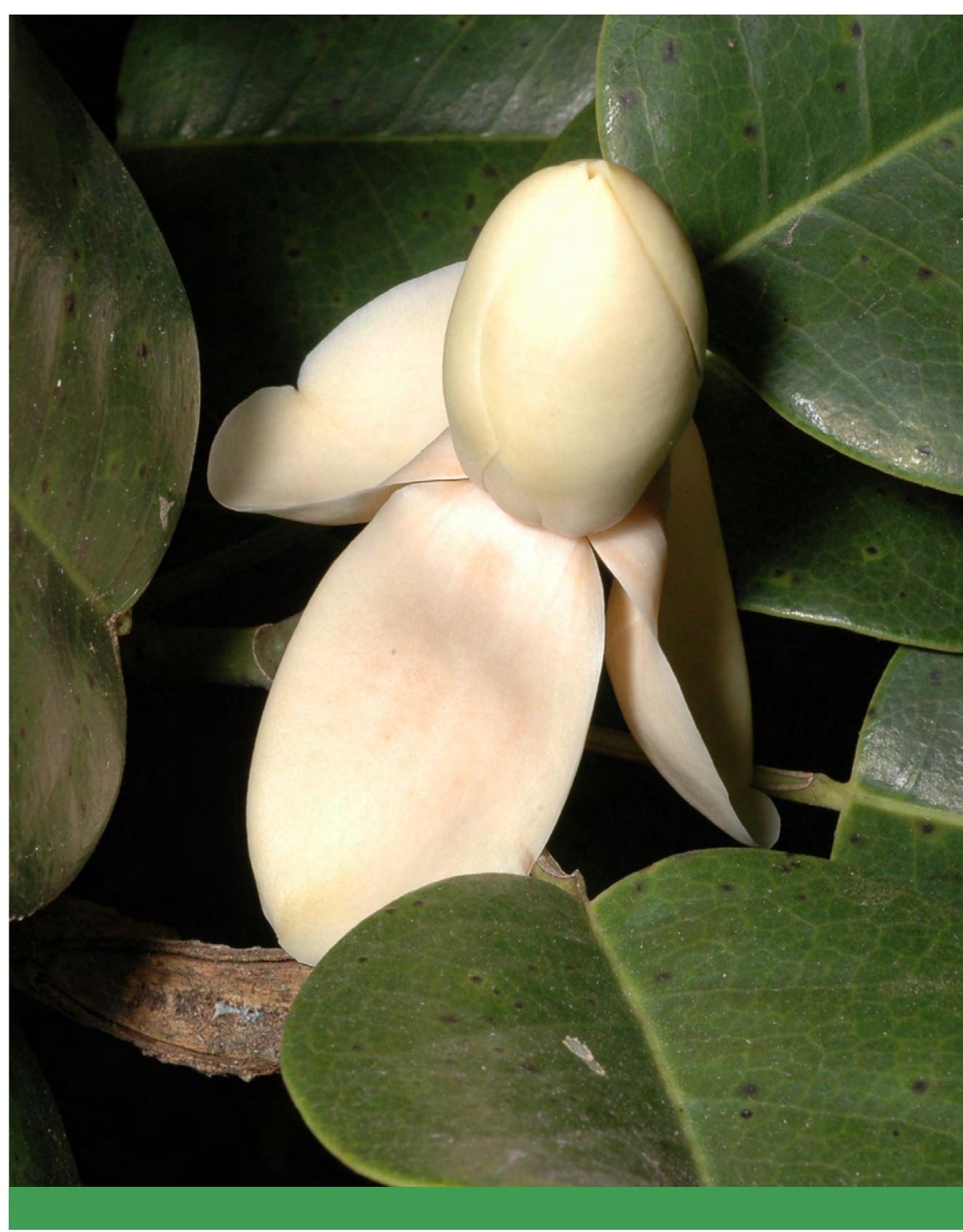

